International Journal of Modern Physics C Vol. 1, No. 4 355-422 (1990)

(C) World Scientific Publishing Company

\title{
QUANTUM LIMITATIONS ON THE STORAGE AND TRANSMISSION OF INFORMATION
}

\author{
JACOB D. BEKENSTEIN \\ Physics Department, Ben Gurion University, Beersheva, 84105 ISRAEL \\ and \\ Racah Institute of Physics, The Hebrew University of Jerusalem \\ Givat Ram, Jerusalem, 91904 ISRAEL * \\ and \\ MARCELO SCHIFFER \\ Insituto de Física Teórica, Rua Pamplona 145, São Paulo, S.P. 01405 BRAZIL
}

Received 7 November 1990

\begin{abstract}
Information must take up space, must weigh, and its flux must be limited. Quantum limits on communication and information storage leading to these conclusions are here described. Quantum channel capacity theory is reviewed for both steady state and burst communication. An analytic approximation is given for the maximum signal information possible with occupation number signal states as a function of mean signal energy. A theorem guaranteeing that these states are optimal for communication is proved. A heuristic "proof" of the linear bound on communication is given, followed by rigorous proofs for signals with specified mean energy, and for signals with given energy budget. And systems of many parallel quantum channels are shown to obey the linear bound for a natural channel architecture. The time-energy uncertainty principle is reformulated in information language by means of the linear bound. The quantum bound on information storage capacity of quantum mechanical and quantum field devices is reviewed. A simplified version of the analytic proof for the bound is given for the latter case. Solitons as information caches are discussed, as is information storage in one dimensional systems. The influence of signal self-gravitation on communication is considerd. Finally, it is shown that acceleration of a receiver acts to block information transfer.
\end{abstract}

Keywords:Information, entropy, coding, communication, quantum channel capacity.

\section{Introduction}

Information, its storage, and its transfer from system to system are all crucial issues in science and technology. They are at the crux of computation. They are connected with the foundations of thermodynamics. Their influence is felt far from the physical sciences. Thus a fundamental aspect in the evolution of life is the ability to store and transmit genetic information at the level of the species. The same is true at the level of society. Human survival rests on the ability of society to acquire and store large quantities of information and transmit it rapidly. Given the importance of the subject, a natural question is whether there are intrisic limitations dictated by the laws of nature on information storage and communication.

Does information take up space? Does it weigh? Can its flux be made arbitrarily large? These related questions must be very old. They evidently have immediate technological bearing. More important, they go right to the heart of the nature of information: is information impalpable, or must it always be associated with material entities? We take it as axiomatic here that there is no such thing as disembodied information, information in the abstract. Information, of whatever kind, must be associated with matter, radiation or fields of some sort. Granted this, the questions raised above can be faced quantitatively.

Some aspects of the query are easy to answer. For example, we know that physical structures cannot travel with speed faster than that of light in vacuum. We infer that information cannot be conveyed from point to point with speed faster than that of light in vacuum. In fact, if one wishes to avoid paradoxes in relativity theory, such as that arising from the fact that phase velocities can sometimes exceed the speed

* Present address. 
of light, one is forced to state the appropriate principle of relativity in the form "information cannot be be propagated at speeds higher than that of light in vacuum".

Of the trio of questions mentioned, that concerning the limitations on the flux of information was the first to be taken up in the wake of developments in communication technology. The primitive answer was the 1930 Hartley-Nyquist law in communication theory ${ }^{1}$ which states that, for a single communication channel, the peak rate of information flow in bits s ${ }^{-1}$ equals to the bandwidth of the channel in Hz. In Shannon's 1948 information theory ${ }^{2}$ the Hartley-Nyquist law is replaced by the famous channel capacity formula, Eq.(5) below, in which (classical) noise in the channel limits the rate at which information can flow through it without incurring errors. This formula has had incalculable influence on communication technology.

Not until the early sixties were quantum generalizations of Shannon's formula proposed. Early expressions of an approximate nature for quantum capacity were proposed by Stern, ${ }^{3}$ Gordon, ${ }^{4,5}$ and Marko. ${ }^{6}$ In 1963 Lebedev and Levitin ${ }^{7}$, starting from thermodynamic considerations, obtained a precise and powerful formula for channel capacity including the effects of both quantum and thermal noise. In the classical limit this formula reduces to Shannon's, while in the noiseless limit it leads back to the estimate of Stein and Gordon [see Eq.(45) below]. Much later Pendry ${ }^{8}$ independently derived the noiseless limit of the LebedevLevitin formula from elegant pure thermodynamic considerations. Gordon's approach ${ }^{5}$ to the quantum channel capacity by combining Shannon's capacity formula with the time-energy uncertainty relation recurs in papers by Bremermann's ${ }^{9-11}$ which, however, led him to a an entirely new result, a linear limit on channel capacity [see Eq.(84) below]. Subjected to heavy criticism, ${ }^{12-14}$ Bremermann's work has been vindicated to some extent, at least isofar as the linear bound can be justified by other means (see Sec.4) The real significance of Bremermann's limit emerges when attention shifts from steady state communication to burst communication. $^{15}$

Interest in fundamental quantum limits on information storage is a later occurrence. It first grew out of developments in black hole thermodynamics. ${ }^{16-18}$ In order to preclude contradictions with the second law of thermodynamics in systems involving black holes and ordinary matter, it turns out necessary to assume that the entropy of an ordinary system is limited in terms of its mass and size. This led one of us ${ }^{16,19}$ to conjecture a quantum upper bound on specific entropy which depends only on the maximum radius of the system in question. Because of the connection between entropy and information, such bound is equivalent to one on the information storage capacity of a system. And combined with causality considerations, this last bound leads ${ }^{12}$ to a limit of the Bremermann type. Much progress has been made in establishing the bound on information storage capacity on the basis of statistical and quantum ideas independent of gravitational physics. ${ }^{13,19,20-23}$

This paper is partly a review of the mentioned developments, and partly a report on a number of related new results obtained lately by us in relation to quantum channel capacity and bound on information storage. It does not give full coverage to all questions related to these subjects. We have not tried to go into details of devices that might implement information storage or communication in such a way as to approach the various bounds mentioned. There are now several good reviews in these areas. ${ }^{24-26}$ We have also steered clear of the subject of bounds on information processing or computation; this large area has also been thoroughly reviewed. ${ }^{14,27}$ In our opinion the relation of the computation process at the elementary level to communication and information storage is not sufficiently understood to allow a review of it to be made at the same level as is possible for the separate areas. For these reasons our list of references on fundamental limits on information is far from complete.

In Sec.2. we review steady state classical and quantum channel capacity theory. New is a detailed development of Lebedev and Levitin's idea of an information theoretic derivation of the quantum capacity for a noisy narrowband channel. In Sec.3 we describe quantum channel capacity theory for burst signals. New here is an accurate analytic approximation to the maximum information possible with occupation number signal states as a function of mean signal energy. We show explicitly that coherent signal states do worse than occupation number states, and prove the theorem that guarantees that occupation number states are optimal in this regard. Sec.4 reviews the linear bound on communication. We give a heuristic "proof" of the bound, followed by rigorous proofs, one applicable to signals with specified mean energy, and the other to signals with given energy budget. New here is a reformulation of the time-energy uncertainty principle in terms of the linear bound, and an discussion of channel capacity for many parallel channels. Sec.5 reviews the evidence for the quantum bound on information storage capacity of quantum mechanical and quantum field systems. We give a new, simplified, version of the analytic proof for the bound in the latter case. Also new here is a discussion of solitons as information caches, and of information storage in one dimensional systems, 
including storage with the help of fluctuations; the bound is shown to be obeyed in both cases. Some aspects of the spacetime view of information are described in Sec.6. New here are discussions of the influence of signal self-gravitation on communication, and of the role of acceleration as a jammer of information transfer.

\section{Limits on Steady State Communication}

The simplest situation regarding communication or information transfer is when steady state obtains. Physically the problem is somewhat analogous to equilibrium thermodynamics, and indeed thermodynamics has played an important role in the development of steady state communication theory. In reviewing it we shall first introduce Shannon's famous information theory, mention his classical channel capacity formula, and pass on to the subject of steady state quantum channel capacity.

\subsection{Shannon's Information Theory}

How to quantify information? Shannon ${ }^{2}$ imagined a system capable of storing information by virtue of its possessing many distinguishable states. Although the system's actual state $a$ is not known a priori, the probabilty for it to occur, $p_{a}$, is assumed known. Shannon sought a measure of the uncertainty about the actual state before the system is examined. He demanded that the uncertainty measure, called entropy and represented as $H\left(p_{1}, p_{2}, \ldots\right)$, satisfy the following requirements:

- $H$ shall be a continuous function of the $p_{a}$.

- When there are $n$ equally probable states, $H$ should monotonically increase with $n$. This is reasonable since more states means more uncertainty.

- Whenever a state $a$ in the original list is found to involve several substates $a_{1}, a_{2}, \ldots$, the original entropy must be augmented by $p_{a} H\left(p_{a_{1}}, p_{a_{2}}, \ldots\right)$. This means that the multiple state is to be treated as a system all by itself, but its entropy is weighed by that state's a priori probability.

Shannon found that the only function satisfying the requirements is

$$
H=-K \sum_{a} p_{a} \ln p_{a}
$$

where $K$ is an arbitrary positive constant, corresponding to different choices of the unit of entropy. The free parameter $K$ can be traded for a free choice of logarithm base, and, therefore, can be set to unity. With logarithms to base 2 the entropy is said to be expressed in "bits" (binary information units). With the natural logarithm it is expressed in "nits" (natural information units), etc. The reader is referred to Shannon's original work ${ }^{2}$ for proof of Eq.(1). In what follows, in line with the "physicist's notation" prevalent in this review, we shall usually compute with the natural logarithm; however, we shall reexpress important results in bits.

When one of the probabilities $p_{a}$ is unity, the entropy vanishes: when the state is known precisely there is no uncertainty. For $\Omega$ possible states, the entropy is maximal when they are all equally likely, and

$$
H_{\max }=\log _{2} \Omega \text { bits. }
$$

The basic idea of information theory is that when the system in question is examined, and the state it is in is fully determined, the amount of information so acquired equals $H$. For example, in old style telegraphy each signal can be either dot or dash with equal probability, so that by Eq.(1) there is one bit of entropy per symbol. When the signal is received and fully identified, one bit of information per symbol is made available. It also follows that if the state is not fully identified, the information obtained is less than the full value of the entropy. Technically the measurement is regarded as imposing constraints which are expressed in terms of conditional probabilities for the various states given the results of the examination. The mean negative logarithm of the conditional probabilities, the conditional entropy, can be proved to be less than the original entropy, ${ }^{2}$ and represents the part of the potential information that was not revealed by examination of the system. We shall express all this in equations in Sec.2.6

Shannon's entropy formula (1) parallels Boltzmann's definition of entropy as used in the proof of the $\mathrm{H}$ theorem. (This explains the use of the name "entropy" and the symbol $H$ in information theory, both suggested to Shannon by von Neumann. ${ }^{2}$ ) Shannon's entropy corresponds to thermodynamic entropy if one equates $K$ with Boltzmann's constant, and uses natural logarithms. Boltzmann's work on statistical 
mechanics already contained germs of the connection between thermodynamic entropy and information. These were amplified later by Szilard in his discussion of the Maxwell demon. ${ }^{28}$ After Shannon's work, Brillouin ${ }^{29}$ worked out in great detail the interrelation of information concepts with thermodynamics. The kinship between Shannon's entropy and the entropy of statistical physics made it possible for Jaynes to use information theory as an axiomatic basis for statistical mechanics. ${ }^{30}$ For a pedagogical treatment the reader is referred to the monograph by Katz. ${ }^{31}$

Shannon's view of information gives up any attempt to ascribe value to information. One bit can stand for rather unimportant information, like specifying the tenth digit of a binary number, or it can represent crucial life saving information, like the bit that triggers an alarm indicating that a patient's heart has stopped. There are alternatives to Shannon's definition which do ascribe a semblance of value to information, for example, algorithmic entropy. ${ }^{32}$ But since most treatments of communication and information storage have had in mind Shannon's definition, we confine our remarks to this context.

\subsection{Shannon's Classical Channel Capacity}

Suppose one wishes to transmit a message through some channel, e.g. the telephone. Typically the message is first converted into a continuous function of time, e.g. the electric current $J(t)$ in the telephone line. If the current lasts time $\tau, J(t)$ is completely specified by the Fourier coefficients

$$
b_{k}=\frac{1}{\tau} \int_{0}^{\tau} J(t) e^{i k \omega t} d t
$$

where $\omega=2 \pi \tau^{-1}$. In practice the signal is restricted to a certain band, an angular frequency range $\Delta \omega$ which may or may not extend down to zero frequency. In the telephone example $\Delta \omega \sim 4 \mathrm{KHz}$. The specification of the physical nature of the signal and of the bandwidth constitute a definition of the communication channel. When $\Delta \omega$ is small compared to the typical frequency in the channel, one calls it a narrowband channel. When $\Delta \omega$ is broader one speaks of a broadband channel. This includes the often discussed case of infinite $\Delta \omega$. Evidently for a narrowband channel all the coefficients with $k \omega>\Delta \omega$ vanish, so that all the information is completely specified by $\tau \Delta \omega / 2 \pi$ complex numbers $b_{k}$ or $n \equiv \tau \Delta \omega / \pi$ real numbers $a_{k}$. Ascribing a probability $p\left(a_{1}, a_{2}, \ldots, a_{n}\right)$ to the set of Fourier components $\left\{a_{1}, a_{2}, \ldots, a_{n}\right\}$, one is in position to calculate the entropy flow rate associated with the signal

$$
\dot{H}=-\frac{1}{\tau} \int p\left(a_{1}, a_{2}, \ldots, a_{n}\right) \ln p\left(a_{1}, a_{2}, \ldots, a_{n}\right) d a_{1} d a_{2} \ldots d a_{n} .
$$

We have glossed here over ambiguities relating to the calculation of entropy from probability densities rather than probabilities for discrete variables. ${ }^{2}$

The above describes an ideal situation. In practice the transmitting channel is affected by noise, e.g. electronic shot noise. Noise has the effect that the received signal differs in a stochastic way from the transmitted one. This limits the receiver's ability to recover the encoded information. In effect the received signal is associated with larger entropy than the transmitted one because the noise has introduced a further measure of uncertainty. It is still possible, in principle, to code the information at the transmitter in such a way that it can all be recovered at the receiver. One may here recall the error correcting codes in effect in intercomputer communication links. However, as proved by Shannon, ${ }^{2}$ elimination of errors upon reception is guaranteed only if the information is not transmitted too fast. In effect every physical channel is ascribed a capacity which represents the maximum rate in bits $s^{-1}$ at which information can be transmitted through it with negligible probability of error. We shall here denote the capacity by $\dot{I}_{\max }$ (standard communication texts usually denote it by $C$ ). Whenever the actual communication rate $\dot{I}$ exceeds $\dot{I}_{\max }$, the difference $\dot{I}-\dot{I}_{\max }$ represents information that will be degraded by errors due to noise.

In Shannon's communication theory the extra uncertainty introduced into the received signal by processes in the channel is quantified by the conditional entropy calculated from the conditional probabilities for various received signal states given that a specific signal state was sent. Evidently the uncertainty here is fully a result of stochastic events in the channel. This conditional entropy is to be substracted from the total entropy of the received signal to obtain the useful information obtainable at the receiver. The channel capacity is the maximum of this last quantity.

Whenever the noise is independent of the transmitted signal, e.g. thermal noise, the conditional entropy is just the entropy constructed from the a priori probabilities for the diverse noise states - the noise entropy. 
Supposing the noise to be Gaussian, and to have power $N$ uniformly distributed over the bandwidth $\Delta \omega$ of the channel (white noise), Shannon obtained from Eq.(4) that the noise entropy $\propto \Delta \omega \log N$. The transmitted signal carries power $P$, so that the received power is $P+N$. The entropy at the receiver is maximized when the total received signal is itself Gaussian. Again from Eq.(4) it is found that the maximum received entropy is $\propto \Delta \omega \log (P+N)$. Subtracting the noise entropy, Shannon obtained the famous classical capacity formula

$$
\dot{I}_{\max }=\left(\frac{\Delta \omega}{2 \pi}\right) \log _{2}\left(\frac{P+N}{N}\right) \text { bits s}^{-1} .
$$

It may be seen that signal-to-noise ratio $P / N$ is the parameter controlling the classical channel capacity. Shannon's theory applies to all signals which may be represented by frequency limited continuous functions of time, i.e., the theory is classical. Shannon's capacity formula successfully describes myriad systems (telephone, fiber optics links, space telemetry, ...). We shall recover it as a limit of the quantum capacity formula for noisy narrowband channels (Sec.2.6)

How may we understand Eq.(5) heuristically? The expression $\Delta \omega / 2 \pi$ is the number of phase space cells passing by a given point per unit time. How much information can be packed in one cell? That depends on the noise which makes it difficult to distinguish one signal from another very close to it in intensity. We can argue that in the presence of noise energy $N \tau$, one can distinguish one level of total signal (signal plus noise) from another only if there are no more than $\frac{(P+N) \tau}{N \tau}$ allowed levels. We interpret this as the number of states available. Maximum information is attained when the states are equally probable, and is given by Eq.(2). We thus get back to Shannon's capacity formula Eq.(5). If the noise is not white, but still Gaussian at each frequency, one can partition the channel into many narrow bands, use Shannon's capacity for each, and convert Eq.(5) into an integral over $\log _{2}[1+P(\omega) / N(\omega)] .^{2}$

Shannon's capacity formula predicts that the capacity diverges logarithmically as the noise is reduced to zero e.g. by cooling the channel for purely thermal noise. This divergence will be seen to disappear in the quantum theory (see Sec.2.5). The Shannon energy cost per bit, $P / \dot{I}_{\max }$, can be written as

$$
\epsilon_{\min }=N \frac{2^{2 \pi \dot{I}_{\max } / \Delta \omega}-1}{\dot{I}_{\max }} .
$$

For given communication rate, $\epsilon_{\min }$ can be reduced arbitrarily by suppress the noise. For thermal noise and low $\dot{I}_{\max }, \epsilon_{\min } \approx k T \ln 2$ [see Eq.(7) below], where $T$ is the absolute temperature of the channel. This reproduces Brillouin's principle ${ }^{29}$ that energy $k T \ln 2$ must be dissipated when a bit of information is acquired in an environment at temperature $T$.

\subsection{Heuristic View of Quantum Channel Capacity}

It is seldom realized that Shannon's classical capacity formula already suggests the form of the quantum capacity formula! To see this assume the noise is thermal. Then, in the classical regime, the noise is white and its power is given by Nyquist's formula ${ }^{29,33}$

$$
N=k T(\Delta \omega / 2 \pi) .
$$

This formula, which merely states that classically each phase space cell carries mean energy $k T$, is accurate for $k T \gg \hbar \omega_{0}$ where $\omega_{0}$ is a typical frequency of the channel. Evidently $\omega_{0}>\Delta \omega / 2$ (the inequality is saturated for a bandwidth extending from zero up to some cutoff if we take $\omega_{0}$ as half the cutoff frequency). The classical regime obtains for $\hbar \omega_{0} \lesssim k T$. Putting the two inequalities in Eq.(7) we get

$$
\Delta \omega \lesssim(4 \pi N / \hbar)^{1 / 2}
$$

This inequality is not a physical restriction on $N$ but merely a guarantee that the classical regime obtains with given $T, \Delta \omega$ and $\omega_{0}$. If the inferred $\Delta \omega$ is not necessarily small, the calculation may nevertheless be justified provided the signal power $P$ is frequency independent also. Then Shannon's capacity formula is valid for a wide band. Substituting Eq.(8) into Eq.(5) we get

$$
\dot{I}_{\max } \lesssim(P / \pi \hbar)^{1 / 2} f(P / N) \text { bits s}^{-1},
$$

where $f(x) \equiv x^{-1 / 2} \log _{2}(1+x)$. Now $f(x)$ has a maximum of $\approx 1.16$ at $x \approx 3.92$; therefore, we find

$$
\dot{I}_{\max } \lesssim 0.65(P / \hbar)^{1 / 2} \text { bits s}^{-1}
$$


What inequality (10) claims is that on the borderline between the classical and quantum regimes, the channel capacity scales as $(P / \hbar)^{1 / 2}$. A complete quantum treatment is necessary to see if this behavior persists deep in the quantum regime. At this point we should mention the common fallacy of substituting the quantum version of Nyquist's noise power Eq.(7) into Shannon's formula in order to derive the quantum channel capacity. ${ }^{5}$ This is incorrect since Shannon's theory describes the signal classically, so that it is not consistent to combine it with a quantum formula for noise power.

\subsection{Quantum Capacity for a Broadband Noiseless Channel}

In the early 1960's Gordon, ${ }^{5}$ gave two early derivations of the quantum channel capacity for a noiseless channel. One was based on the time-energy uncertainty relation, a very popular though flawed approach which confuses the time entering into the principle with the duration of the signal. The second approach, already criticized in Sec.2.3., combined the classical Shannon capacity formula with Nyquist's quantum noise formula. Neither gave the correct coefficient in the quantum channel capacity, Eq.(16) below, but both gave the correct dependence $\dot{I}_{\max } \propto(P / \hbar)^{1 / 2}$. Stern ${ }^{3}$ and Marko ${ }^{6}$ had a similar measure of success by other approaches. Before describing the full thermodynamic derivation of the quantum capacity due to Lebedev and Levitin, ${ }^{7}$ which includes the effects of thermal noise, we shall review the more recent thermodynamic derivation of Pendry ${ }^{8}$ which deals specifically with a noiseless channel. It illustrates well two important issues: the difference between boson and fermion channels, and the insensitivity of the channel capacity to dispersion.

Pendry's focuses on the channel and the carrying field, rather than on the process of detection. His description of signals, unlike Shannon's, is a quantum one: each possible signal is represented by a particular quantum state of the field, e.g. a particular set of occupation numbers for the various propagating modes in the channel. Pendry assumes uniformity of the channel in the direction of propagation, which allows him to label modes by momentum $p$. He allows dispersion so that a quantum of momentum $p$ has some energy $\varepsilon(p)$. Then the propagation velocity of the quanta is the group velocity $v(\varepsilon)=d \varepsilon(p) / d p$.

The basic assumption is that $\dot{I}_{\max }$ can be identified (apart from units) with the unidirectional thermodynamic entropy current that the channel carries in a thermal state. This hails back to the idea that in a thermal state the entropy in each mode is maximal. Of course in the thermal state there is no net flow of entropy, but all modes moving in a definite sense along the channel do carry an entropy current. It is assumed to be maximal.

Now the entropy $s(p)$ of a boson mode of momentum $p$ in thermal equilibrium at temperature $T$ is $^{34}$

$$
s(p)=\frac{\varepsilon(p) / k T}{e^{\varepsilon(p) / k T}-1}-\ln \left(1-e^{-\varepsilon(p) / k T}\right) .
$$

The entropy current in one direction is thus

$$
\dot{H}=\int_{0}^{\infty} s(p) v(\varepsilon) d p / 2 \pi \hbar,
$$

where $d p / 2 \pi \hbar$ is the number of modes per unit length in the interval $d p$ which go by in one direction. This factor, when multiplied by the group velocity, gives the unidirectional current of modes.

After an integration by parts on the second term coming from (11), we can cast the last result into the form

$$
\dot{H}=\frac{2}{k T} \int_{0}^{\infty} \frac{\varepsilon(p)}{e^{\varepsilon(p) / k T}-1} \frac{d \varepsilon(p)}{d p} \frac{d p}{2 \pi \hbar} .
$$

The first factor in the integrand is the mean energy per mode, so that the integral represents the unidirectional power $P$ in the channel:

$$
\dot{H}=2 P / k T \text {. }
$$

The integral in Eq.(13) is evaluated by cancelling the two differentials $d p$ and assuming the energy spectrum is single valued and extends from 0 to $\infty$. Then the form of the dispersion relation $\varepsilon(p)$ does not enter, and Pendry's result is

$$
P=\pi(k T)^{2} / 12 \hbar .
$$

The last and crucial step is to eliminate $k T$ between the expressions for $\dot{H}$ and $P$. Multiplying by $\log _{2} e$ to convert thermodynamic units to bits one has

$$
\dot{I}_{\max }=(\pi P / 3 \hbar)^{1 / 2} \log _{2} e \text { bits s} ~^{-1}
$$


which is the noiseless quantum channel capacity. (The analogous calculation for Fermi statistics gives a capacity smaller by a factor $\sqrt{ } 2$. Pendry ${ }^{8}$ actually quotes the same capacity as for bosons, but this is because he considers the contributions of both particles and holes in a solid state communication channel). Henceforth we refer to Eq.(16) simply as Pendry's formula; it must be borne in mind, however, that this result appeared in the earlier work of Lebedev and Levitin ${ }^{7}$, and in approximate form in Refs.3, 5 and 6 .

Instead of Eq.(6) of Shannon's theory we have here the energy cost per bit

$$
\epsilon_{\min }=3 \hbar \pi^{-1}(\ln 2)^{2} \dot{I}_{\max }
$$

(For a fermionic channel the energy cost per bit is a factor $\sqrt{ } 2$ larger.) Whereas the energy cost per bit in classical theory rises exponentially with $\dot{I}_{\max }$, the quantum energy cost per bit grows only linearly.

It is somewhat surprising that the channel capacity is independent, not only of the form of the mode velocity $v(\varepsilon)$, but also of its scale. Phonon channel capacity is as large as photon channel capacity despite the difference in speeds. Why? Although phonons convey information at lower speed, the energy of a phonon is proportionately smaller than that of a photon in the equivalent mode. When the capacity is expressed in terms of the energy flux, it thus turns out to involve the same constants. We may also offer the trivial comment that the capacity for massive bosons must be lower than Eq.(16) since part of the energy is locked in rest mass, and thus the range of modes available for information carrying is smaller than in the massless case.

\subsection{Broadband Channel Subject to Thermal Noise}

Lebedev and Levitin's derivation of the quantum channel capacity, ${ }^{6}$ like Pendry's much latter one, was a thermodynamic derivation. Unlike Pendry's approach, this one focuses on the process of detection. Although Lebedev and Levitin were thinking of electromagnetic transmission, their results apply to any single channel carrying a Bose field (one polarization and fixed wave vector electromagnetic, fixed wave vector acoustic, ...), and they can easily be extended to channels carrying fermion fields. For mathematical convenience the signal is regarded as periodic with very long period $\tau$. Therefore, the angular frequencies present are

$\omega_{j}=2 \pi j \tau^{-1}$. Again each possible signal state is regarded as represented by a specific set of occupation numbers of the various modes $\omega_{j}$. The whole communication system is regarded as subject to thermal noise characterized by a temperature $T_{1}$.

The detector is idealized as a collection of harmonic oscillators, one for each $\omega_{j}$. The thermal energy of the oscillators before any signal is received follows from Planck's formula

$$
E_{1}=E\left(T_{1}\right) \equiv \sum_{j=1}^{\infty} \frac{\hbar \omega_{j}}{e^{\hbar \omega_{j} / k T_{1}}-1} .
$$

The thermodynamic entropy in the oscillators is

$$
H_{1}=H\left(E_{1}\right) \equiv \int_{0}^{E_{1}} \frac{d E^{\prime}}{T^{\prime}}=\int_{0}^{T_{1}} \frac{1}{T^{\prime}} \frac{d E\left(T^{\prime}\right)}{d T^{\prime}} d T^{\prime} .
$$

If the signal carries power $P$, the energy of the oscillators is changed to $E_{1}+P \tau$ upon reception of a full period of signal. The signal arrives in a particular (pure) quantum state, and thus brings no entropy with it, so that the detector entropy is still $H_{1}$. It is clear that this is below the maximum entropy possible with the new energy $E_{2} \equiv E_{1}+P \tau$. According to Brillouin's principle, ${ }^{29}$ the deficit is a measure of the maximum information $I_{\max }$ that can now be contained in the detector. This principle is, of course, merely a variant of Shannon's information principle stated in Sec.2.1. Accordingly one can write

$$
I_{\max }=k^{-1}\left[H\left(E_{2}\right)-H\left(E_{1}\right)\right] \log _{2} e \text { bits, }
$$

where Boltzmann's constant $k$ transforms from thermodynamic units to nits, and $\log _{2} e$ from nits to bits. The capacity $\dot{I}_{\max }$ follows by dividing $I_{\max }$ by $\tau$.

By now it must be clear that the maximum information transmitted corresponds to the situation when $H\left(E_{2}\right)$ is maximal, i.e., for a thermal state characterized by the formal temperature $T_{2}$ defined by $E\left(T_{2}\right)=$ $E_{2}$. Keeping in mind that the noise is also thermal, it follows from Eqs.(19)-(20) that

$$
\dot{I}_{\max }=\int_{T_{1}}^{T_{2}} \frac{1}{k T^{\prime}} \frac{d W\left(T^{\prime}\right)}{d T^{\prime}} d T^{\prime} \log _{2} \text { e bits } \mathrm{s}^{-1}
$$


Here $W(T)$ is just $E(T) / \tau$, the thermal power issuing from the channel when it is at temperature $T$. Substituting $\omega_{j}=2 \pi j \tau^{-1}$ into Eq.(18) and passing to the continuum limit by means of the rule $\tau^{-1} \sum \rightarrow$ $\int 2 \pi^{-1} d \omega$ one gets

$$
W(T)=\frac{\pi(k T)^{2}}{12 \hbar},
$$

which is equivalent to Pendry's result Eq.(15).

This result is useful in two ways. From $E_{2}=E_{1}+P \tau$ it is evident that $W\left(T_{2}\right)=W\left(T_{1}\right)+P$. From Eq.(22) it now follows that

$$
T_{2}=T_{1}\left[1+\frac{12 \hbar P}{\pi\left(k T_{1}\right)^{2}}\right]^{1 / 2}
$$

In addition it follows from substituting (22) in (21) that

$$
\dot{I}_{\max }=\frac{\pi k}{6 \hbar}\left(T_{2}-T_{1}\right) \log _{2} e \text { bits s}^{-1}
$$

Elimination of $T_{2}$ between (23) and (24) finally gives the Lebedev-Levitin capacity for a noisy channel at temperature $T_{1}$

$$
\dot{I}_{\max }=\frac{\pi k T_{1}}{6 \hbar}\left\{\left[1+\frac{12 \hbar P}{\pi\left(k T_{1}\right)^{2}}\right]^{1 / 2}-1\right\} \log _{2} e \text { bits s}^{-1} .
$$

In the classical (or low signal power) limit $P \hbar /\left(k T_{1}\right)^{2} \ll 1$, this formula reduces to

$$
\dot{I}_{\max }=\left(P / k T_{1}\right) \log _{2} e \operatorname{bits~s}^{-1},
$$

which coincides with the low signal-to-noise limit of Shannon's capacity formula (5) when the noise power $N$ is given in terms of $T_{1}$ by Nyquist's formula (7). [Strictly speaking one has to assume a white noise spectrum in order to compare the Shannon formula with the wideband result, Eq.(26).] In the quantum (or high signal power) limit, Lebedev and Levitin's formula goes over to Pendry's Eq.(16).

The energy cost per bit $P / \dot{I}_{\max }$ computed from Eq.(25) can be cast, after some algebra, into the convenient form

$$
\epsilon_{\min }=\left(k T_{1}+\frac{3 \hbar}{\pi} \dot{I}_{\max }\right) \ln 2 .
$$

In this formula the classical and quantum contributions are neatly additive. The first term is Brillouin's classical energy cost per bit; the second, clearly the energy cost per bit arising from quantum fluctuations (some say "quantum noise"), coincides with Eq.(17) for Pendry's noiseless quantum channel.

The importance of the channel capacity formula, Eq.(25), should not be overstated. It is an upper bound on the channel capacity only if the noise is thermal. This is because the thermal distribution maximizes entropy rate for given power. Thus for nonthermal, e.g. Poisson, noise we would substract a smaller number in Eq.(20), and would get a larger capacity than inferred from Eq.(25) with $T_{1}$ replaced by noise power $N$ according to Eq.(22). But since it is impossible to exceed the noiseless channel capacity Eq.(16), if we wish to be noncommittal about the nature of the noise, we should write

$$
\left(\frac{\pi P}{3 \hbar}\right)^{1 / 2} \frac{[1+P / N]^{1 / 2}-1}{(P / N)^{1 / 2}} \log _{2} e \text { bits s}^{-1} \leq \dot{I}_{\max } \leq\left(\frac{\pi P}{3 \hbar}\right)^{1 / 2} \log _{2} e \text { bits s}^{-1}
$$

\subsection{Narrowband Channel Subject to Noise}

Notwithstanding the conceptual simplicity of the foregoing discussion, in practice communication channels are narrowband channels. In attempting to deal with the latter, it is most instructive to treat the flow of information through a single mode of the channel. Because usually the separate modes are decoupled, the result for a narrowband channel will follow from summation over modes. Rather than follow the thermodynamic approach of Lebedev and Levitin, we emphasize here the information-theoretic approach that may be used to deal with noise (this method was also alluded in Lebedev and Levitin's paper).

Let the input signal contain a mean number of quanta $\bar{m}$. We associate with it a probability distribution for the number of quanta $p_{i}(m)$. Having negotiated the channel, the signal enters the receiver which is modeled as an harmonic oscillator of frequency $\omega$. Due to noise the oscillator is initially in a mixed state 
characterized by the mean occupation number $\bar{\ell}$. Let us parametrize the noise by the parameter $\alpha$ defined by

$$
\bar{\ell}=\frac{1}{e^{\alpha}-1} .
$$

In case the noise is thermal Planck's law gives $\alpha=\hbar \omega / k T$ where $T$ is the temperature. The oscillator's entropy may be calculated by looking for that probability distribution $r(\ell)$ which maximizes the Shannon entropy $H=-\sum_{\ell} r(\ell) \ln r(\ell)$ subject to the constraint that the mean number of quanta be $\bar{\ell}$. This happens to be the exponential (thermal) distribution

$$
r(\ell)=\left(1-e^{-\alpha}\right) e^{-\alpha \ell}
$$

The corresponding noise entropy is

$$
H_{n}=\frac{\alpha}{e^{\alpha-1}}-\ln \left(1-e^{-\alpha}\right) .
$$

Upon reception of the signal the mean number of quanta in the oscillator goes up to [see Eq.(29)]

$$
\bar{\ell}=\frac{1}{e^{\alpha}-1}+\bar{n}_{i}
$$

How much information is now contained in the receiver? Since the number of quanta $n$ in it is partly a result of noise, we cannot identify the quantity of information with the entropy $H_{o}$ of the output signal as calculated from its probability distribution $p_{o}(n)$. Neither is the entropy of the initial signal $H_{i}$ the correct quantity; it did quantify the information that could be borne by the signal, but this information has since been adulterated by noise.

The procedure for dealing with this situation was outlined by Shannon. ${ }^{2}$ There is a joint probability distribution $p_{o, i}(n, m)$ for input and output numbers of quanta which supplies a complete statistical description of the noisy system. From it we can compute the two marginal probability distributions, one, $p_{i}(m)$, by summing out $n$, and a second one, $p_{o}(n)$, by summing out $m$, as well as two conditional distributions. One

$$
p_{o \mid i}(n \mid m) \equiv p_{o, i}(n, m) / p_{i}(m)
$$

stands for the probability of $n$ quanta in the detector given that $m$ were sent. The second,

$$
p_{i \mid o}(m \mid n) \equiv p_{o, i}(n, m) / p_{o}(n),
$$

gives the probability that $m$ quanta were sent given that the detector contains $n$.

There is an entropy for each of these distributions. The generic definition is

$$
H_{a} \equiv \sum_{n, m} p_{o, i}(n, m) \log p_{a}(\text { indexes relevant to } a)
$$

where $a$ can stand for $i, o,(o, i),(i \mid o)$ or $(o \mid i)$. The following identities ${ }^{2}$ are easily verified:

$$
H_{o, i}=H_{i}+H_{o \mid i}=H_{o}+H_{i \mid o}
$$

Shannon noted that $H_{i \mid o}$, the conditional entropy of the input when the output is known, must represent the extra uncertainty introduced by the noise which hinders reconstruction of the initial signal even when the output is known. He thus interpreted $H_{i}-H_{i \mid o}$ to be the useful information $I$ that can be recovered from the output signal (by means of appropriate coding and decoding) in the face of noise. Another way to understand this is to rewrite this definition with help of Eq.(36) as

$$
I=H_{o}-H_{o \mid i} .
$$

We can think of $H_{o \mid i}$, the uncertainty in the output for given input, as the effect of the noise. Therefore, it is to be subtracted from the full entropy of the output $H_{o}$ to get the uncertainty asociated with the signal itself. 
Now in the case being considered, the noise is independent of the signal and described by distribution (30). Therefore, $p_{o, i}(n, m)=p_{i}(m) r(n-m)$. It follows from (33) that $p_{o \mid i}(n \mid m)=r(n-m)$ so that (37) gives

$$
I=H_{o}-\sum_{n, m} p_{i}(m) r(n-m) \log r(n-m) .
$$

The sum over $n \geq m$ for fixed $m$ gives just $H_{n}$, the noise entropy. For thermal noise it is given by Eq.(31). Summation over $m$ just multiplies by the normalization factor 1 . Thus

$$
I=H_{o}-H_{n}
$$

It should be clear from the foregoing that Brillouin's principle is only valid in the case that signal and noise are statistically independent. For example, if the "noise" were due to stimulated emission which is influenced by the incoming signal, Eq.(39) would not apply.

We must still maximize $I$ over the distribution $p_{o}(n)$ subject to the mean number of quanta $\bar{\ell}$ given by Eq.(32). In analogy with the discussion leading to Eq.(30) we find that $H_{o}$ is maximized for an exponential distribution like (30) but with a parameter $\beta$ determined by

$$
\frac{1}{e^{\beta}-1}=\frac{1}{e^{\alpha}-1}+\bar{n}_{i} .
$$

The maximal entropy is the analog of (31); therefore,

$$
I_{\max }=\frac{\beta}{e^{\beta}-1}-\ln \left(1-e^{-\beta}\right)-H_{n} .
$$

We know that for thermal noise $H_{n}$ takes on its maximal value, Eq.(31), for given mean number of noise quanta $\bar{\ell}$. This means that $I_{\max }$ is actually smaller than than for any other kind of noise with the same $\bar{\ell}$. Thus

$$
\frac{\beta}{e^{\beta}-1}-\ln \left(1-e^{-\beta}\right)-\frac{\alpha}{e^{\alpha}-1}+\ln \left(1-e^{-\alpha}\right) \leq I_{\max } \leq \frac{\beta}{e^{\beta}-1}-\ln \left(1-e^{-\beta}\right),
$$

which is the one-mode analog of (28).

Recall that this is the information per mode. Now if the channel in question has bandwidth $\Delta \omega$, a total of $\Delta \omega / 2 \pi$ modes reach the receiver per unit time. Also, we may define the differential power $P_{\omega}$ as the energy per unit time per unit circular frequency. Clearly since each quantum carries energy $\hbar \omega, \bar{n}_{i}=2 \pi P_{\omega} / \hbar \omega$. Making these substitutions in (31), (40)-(41) we get for a narrowband channel with thermal noise

$$
\begin{gathered}
\dot{I}_{\max }=\frac{\Delta \omega}{2 \pi}\left\{\ln \left[1+\frac{2 \pi P_{\omega}}{\hbar \omega}\left(1-e^{-\hbar \omega / k T}\right)\right]+\left(\frac{2 \pi P_{\omega}}{\hbar \omega}+\frac{1}{e^{\hbar \omega / k T}-1}\right) \times\right. \\
\left.\ln \left[1+\frac{e^{\hbar \omega / k T}-1}{\frac{2 \pi P_{\omega}}{\hbar \omega}\left(e^{\hbar \omega / k T}-1\right)+1}\right]-\frac{\hbar \omega / k T}{e^{\hbar \omega / k T}-1}\right\} \log _{2} e \text { bits s }^{-1} .
\end{gathered}
$$

A formula of this form was first given by Gordon ${ }^{4}$, and was later rederived by Lebedev and Levitin ${ }^{7}$ by the thermodynamic method reviewed in Sec.2.4.

The classical limit $(\hbar \omega \ll k T)$ of Eq. $(43)$ is

$$
\dot{I}_{\max } \approx \frac{\Delta \omega}{2 \pi} \log _{2}\left(1+\frac{2 \pi P_{\omega}}{k T}\right) \text { bits s}^{-1},
$$

which coincides with Shannon's capacity formula Eq.(5) when one uses the Nyquist formula for thermal noise Eq.(7). However, the Shannon formula for arbitrary noise cannot be gotten from (41) in any simple way.

In the extreme quantum limit $(k T \ll \hbar \omega)$ (or in the noiseless case) we get

$$
\dot{I}_{\max } \approx \frac{\Delta \omega}{2 \pi}\left[\log _{2}\left(1+\frac{2 \pi P_{\omega}}{\hbar \omega}\right)+\frac{2 \pi P_{\omega}}{\hbar \omega} \log _{2}\left(1+\frac{\hbar \omega}{2 \pi P_{\omega}}\right)\right] \text { bits s}^{-1},
$$

a formula previously given by Stern ${ }^{3}$, Gordon ${ }^{4}$, Lebedev and Levitin ${ }^{7}$, Yamamoto and Haus, ${ }^{24}$ and Takahashi ${ }^{25}$ among others. The two terms in brackets have interesting interpretations. ${ }^{24}$ The first dominates at large power or for occupation number large compared to unity. It tells us that the information delivered per mode 
is the logarithm of the mean occupation number plus one. We may call this the wave contribution because it dominates whenever the signal can be treated as a wave. The second term dominates when the occupation number is small compared to unity. Since $\Delta \omega P_{\omega} / \hbar \omega$ is just the rate at which quanta arrive, it attributes to each photon information equal to the logarithm of one plus the number of modes per photon. Plainly the corpuscular aspect of the signal is manifested here.

Yamamoto and Haus $^{24}$ have discussed the information per quantum and the energy cost per bit for the narrowband channel in various limits. For general $\hbar \omega / k T$ in the low signal power case, $2 \pi P_{\omega} \ll \hbar \omega$, one has $\epsilon_{\min } \approx k T \ln 2$, which coincides with Brillouin's term in Eq.(27) for the broadband channel. In the noiseless channel case the energy cost per bit diverges as $P_{\omega} \rightarrow 0$ like $-\log \left(P_{\omega}\right)$. We shall have more to say about this in Sec.3.4.

We still have to settle one question. In order to reach the peak communication rate, how should one code the signal? The mathematical question is what should be the adopted probability distribution $p_{i}(m)$ for the signal? It can be found with help of the following theorem.

Theorem 1. When an integer-valued variable $\ell$ with exponential distribution $p(\ell)=\left(1-e^{-\alpha}\right) e^{-\alpha \ell}$ is added to an independent integer-valued variable $m$ with distribution $Q(m)$, and there results a variable with exponential distribution with parameter $\beta$, the distribution $Q(m)$ must be a modified exponential one:

$$
Q(m)=\frac{1-e^{-\beta}}{1-e^{-\alpha}} e^{-\beta m} \times \begin{cases}1 & \text { if } m=0 \\ 1-e^{\beta-\alpha} & \text { if } m \geq 1 .\end{cases}
$$

Proof. The proof is given in Appendix A.

We now identify the noise in the receiver with the exponentially distributed variable $\ell$, and $p_{o}(n)$ with the exponential distribution with parameter $\beta$. The theorem tells us that $p_{i}(m)$ must be identified with $Q(m)$ of Eq.(46): the signal distribution must be chosen as modified exponential with the parameters $\alpha$ and $\beta$ defined by Eqs.(29) and (40).

Thus far our discussion has been based on occupation number states as signal states. But, of course, there are other choices, e.g. coherent states, in-phase squeezed states, photon number squeezed states, ... As shown in Sec.3.5. the maximum communication rate is lower when coherent states are used. Yamamoto and Haus $^{24}$ and Saleh and Teich ${ }^{26}$ have analyzed the implementation of quite a variety of quantum states in communication by means of quantum optical techniques. They find that the maximal communication rate does depend on the type of state as well as the type of measurement performed by the receiver, but conclude that the capacity (45) cannot be exceeded. This feeling can be formalized; a general theorem to this effect is proved in Sec.3.6.

\section{Limits on Burst Communication Through Noiseless Channels}

A priori there is no guarantee that the previous results Eqs.(16), (25) and (43) apply to signals of finite duration. This is because all of them can be obtained by thermodynamic arguments, and thermodynamics is usually applicable only in equilibrium. This suggests that the mentioned capacities are, strictly speaking, valid only for steady state communication, namely communication using signals of very long duration where the information and energy flow can be construed as in steady state. So we may ask, what is the capacity for burst communication?

Already Shannon ${ }^{2}$ worried about departures from the simple capacity formula (5) when the power is not steady, and worked out some bounds on capacity expressed in terms of mean or instantaneous power. Interest in the quantum capacity for nonsteady state communication developed rather late. We have already mentioned Bremermann's heuristic formula ${ }^{9-11}$ [see (84) below] which purports to bound the capacity in terms of the energy available to the signal. Bremermann's arguments, and Bekenstein's much later one, ${ }^{12}$ which gave a similar formula, were based on specific models. Before getting into all that it is useful, following Ref.15, to use general arguments to write down a bound on communication via a single channel when steady state does not hold.

\subsection{General Form of Bound on Burst Communication}

Guided by the results reviewed in Sec.2, we take the view that the only specific signal parameters are duration $\tau$ and energy $E$. The rest, e.g. polarization of electromagnetic signals, wave vector direction, etc. , are descriptive of the channel. Thus different polarizations, quanta species, etc. are to be associated with separate channels: unpolarized light, even if monochromatic and perfectly collimated, is regarded as propagating through two channels, say left and right circularly polarized. And an hypothetical communication 
system involving monochromatic collimated beams of neutrinos will entail one channel for each neutrino species (flavor). This precaution is useful in removing energy degeneracies in the subsequent treatment.

How is the maximum information $I_{\max }$ a signal may bear related to $E$ and $\tau$ ? Since information is dimensionless, $I_{\max }$ must be a function of dimensionless combinations of $E$, $\tau$, channel parameters and fundamental constants. We exclude channels which transmit massive quanta, e.g. electrons, because rest mass is energy in a form not useful for communication, so that the strictest limits on capacity and the energy cost per bit are expected for massless signal carriers. Hence Compton lengths do not enter into the argument. Also in order to maximize the information flux we focus on broadband channels, and exclude any frequency cutoff and its associated length. If we also exclude the gravitational constant from the argument on the grounds that gravity can only bring about small effects (see Sec.6.2. for a deeper argument) there is a single dimensionless combination of the parameters that can enter: $\xi=E \tau / \hbar$. It follows that

$$
I_{\max }=\Im(E \tau / \hbar),
$$

where $\Im(\xi)$ is some nonnegative valued function characteristic of the channel. We call it the characteristic information function or CIF.

The reader may find it surprising that the ratio $c_{s} / c$, where $c_{s}$ is the propagation speed of signals, e.g. the speed of sound, was not considered in our argument. Obviously the ratio, if different from unity, is a property of the channel, not of individual signals. Therefore, it is regarded as determining the form of the one-argument function $\Im(\xi)$. It will soon become clear that in many cases $c_{s} / c$ does not appear at all in the CIF. In fact Pendry's argument reviewed in Sec.2.4. makes it clear that signal speed becomes irrelevant in the limit of long signal duration or steady state.

Let us check formula (47). Consider steady state communication. Because of the statistically stationary character of the signal, it should be possible to infer the peak communication rate by considering only a finite section of the signal bearing information $I_{\max }$ and energy $E$. It should matter little how long a stretch in $\tau$ is used so long as it is not short. This can only be true if $\dot{I}_{\max } \equiv I_{\max } \tau^{-1}$ is fully determined by the power $P \equiv E \tau^{-1}$. This is consistent with $I_{\max }=\Im(\xi)$ only if $\Im(\xi)=B \sqrt{ } \xi$ where $B$ is a constant; only then does $\tau$ cancel out. It follows that $\dot{I}_{\max }=B(P / \hbar)^{1 / 2}$ which is precisely the Pendry formula (16). The argument is, however, too general to say anything about the value of $B$ which depends sensitively on the channel's parameters.

The dividing line between steady state communication, and communication by means of very long signals is not sharp. This suggests that long signals must also obey a Pendry type formula, albeit approximately. Indeed, long ago Marko ${ }^{6}$ proposed that $I_{\max } \propto(E \tau / \hbar)^{1 / 2}$ for long duration signals. As we shall see in Sec.3.4., for $\xi=E \tau / \hbar \gtrsim 100, \Im(\xi) \rightarrow B \sqrt{ } \xi$. For $E \tau / \hbar \lesssim 100$ signal end effects become significant, and $\Im(\xi)$ departs from the form $\sqrt{ } \xi$.

\subsection{Signals With Specified Mean Energy}

The energy $E$ which enters in Eq.(47) is subject to various interpretations. Is it the precise energy of the signal, the mean energy (mean with respect to a probability distribution), or the maximum available energy? In this and the following subsections we consider the implications of specifying the signal by its mean energy $\bar{E}$. The case when $E$ is the maximum available signal energy is the subject of Sec.4.

In order for a signal to be able to carry information, there must be various possible signal states. Each state $a$ has its own well defined energy $E_{a}$ and is assigned an a priori probability $p_{a}$ satisfying $\sum_{a} p_{a}=1$. The mean energy is defined by

$$
\bar{E}=\sum_{a} p_{a} E_{a}
$$

What is the capacity for signals with specified $\bar{E}$ ? Evidently we are called to maximize the Shannon entropy Eq.(1) subject to the normalization constraint, Eq.(48), and any other constraints deriving from the nature of the problem. If there is noise, one must deal with it along the lines reviewed in Sec.2.6. For simplicity we focus here on noiseless channels. Are there any other relevant constraints, for example, those imposed by the nature of the reception?

Clearly the formal distribution $p_{a}$ is physically relevant if all states $a$ can be detected and distinguished by the receiver. If several states can be confused, the distribution should assign them equal probabilities, and this should be taken into account in the maximization process. Here we shall be concerned with the more profound question of whether the vacuum signal state, e.g. the "no photon" signal state in an electromagnetic 
channel, can be used to signal. Can the receiver distinguish between a situation where a signal has arrived with all the relevant modes in the vacuum (or ground) state and one in which no signal was received? Only if the answer is affirmative is it appropriate to assign nonvanishing probability to the vacuum signal state. The question comes up because there are situations where even if undetectable, the vacuum can still be used for signaling.

For example, in a man-made channel transmitting a train of signals at equally spaced intervals, the absence of energy in a particular time interval (not the first or last) implies that that signal is in the vacuum state. The embedding of a signal in a series is not the only way to use the vacuum. Let two friends A and $\mathrm{B}$ agree that if A passes his exam, he will phone B between 2 and 3 p.m. If B's phone fails to ring in that period, he has acquired a bit of information (A has failed), by receiving the vacuum state of the signal. If in a scattering experiment at an accelerator no relevant events are detected, information is obtained (upper bound on the cross section) by means of the vacuum state of the signal. What is common to these examples is that the signal is anticipated by virtue of its being part of a structure (series), by prior agreement (phone, if you pass), or by causality considerations (no scattering expected, unless accelerator has been is on). A signal of this sort is aptly termed a heralded signal. For heralded signals the vacuum state, even if not directly detectable, can be put to use in signaling just as any other state.

Consider now a signal whose arrival time is unanticipated. The observation of the recent supernova outburst in the Large Magellanic Cloud, or the beta decay of a particular radioactive nucleus provide two examples of events that could not have been foreseen by the observer. Here the vacuum state cannot be inferred by elimination since the receiver does not know when to expect it, and so cannot carry out measurements, e.g. counting photons with a photomultiplier or measuring the momentum of a beta particle. Hence such a signal, if detected, is always received in a nonvacuum state: the signal heralds itself. We shall call such signals self-heralding. We see that steady state communication is based on heralding signals since a sudden absence of a signal during a very long transmission can be used to convey information. It is also clear that burst communication at unanticipated times must be based on self-heralding signals.

\subsection{Generic Properties of the CIF}

When we consider self-heralding signals, the vacuum signal state must be excluded from the list of signal states. Formally this means $p_{\text {vac }} \equiv \operatorname{Pr}\left(E_{a}=0\right)=0$. Let us maximize the Shannon entropy (1) over the nonvacuum states subject to normalization of probability and the condition (48). The result can be written in a form applicable to both types of signals:

$$
p_{a}=C \times\left\{\begin{array}{cc}
e^{-\mu E_{a}} & E_{a} \neq 0 \\
1-\zeta & E_{a}=0
\end{array}\right.
$$

where $\zeta=0$ for heralded signals, and $\zeta=1$ for self-heralding ones. Although other values of $\zeta$ seem to have no physical relevance, all the calculations to follow are unified if we keep $\zeta$ general.

Let us define the "partition function":

$$
Z \equiv \sum_{a} e^{-\mu E_{a}}
$$

where $\mu$ is a parameter analogous to inverse temperature in statistical mechanics. The normalization constant is now given by

$$
C=(Z-\zeta)^{-1}
$$

When $\zeta \neq 0$ this is a bit different from the statistical mechanics result. As in statistical mechanics, the expression for the mean energy can here be cast into the form

$$
\bar{E}=\partial \ln C / \partial \mu,
$$

which determines $\mu$ in terms of the prescribed $\bar{E}$.

The calculation of $I_{\max }$ from the Shannon entropy corresponding to distribution (49) gives

$$
I_{\max }=\mu \bar{E} \log _{2} e-\log _{2} C-C(1-\zeta) \log _{2}(1-\zeta) \text { bits. }
$$

Formally the last term vanishes for both $\zeta=1$ and $\zeta=0$. Of course, this does not mean that self-heralding and heralded signals bear identical information because, for given $\bar{E}$, the two will have different $\mu$ 's [see(52)]. Eqs. (52)-(53) give, in parametric form, $I_{\max }(\bar{E})$, and thus determine the form of the CIF. 
Several properties of the CIF follow immediately. For example, differentiating (53) with respect to $\bar{E}$ and using (52) we get for $\zeta=0,1$ that $\partial I_{\max } / \partial \bar{E}=\mu$. Since $\mu$ must be positive (otherwise $Z$ would diverge and $C$ vanish), we find that $\Im(\xi)$ is always an increasing function ( $\tau$ is a fixed parameter in the present exercise). This conclusion also holds formally for $0<\zeta<1$.

A look at (50)-(51) shows that in the limit of small $\mu$ (large $\bar{E}$ or $\xi$ ), the partition function overwhelms $\zeta$. Thus at large argument the CIF's for heralded and self-heralding signals must merge. It will become clear that they go over into the CIF associated with the Pendry formula, $\Im(\xi) \propto \sqrt{ } \xi$ (see Sec.3.4.).

Taking the second derivative of Eq.(53), and observing that necessarily $\partial \bar{E} / \partial \mu<0$ by the analogy between $\mu$ and inverse temperature, we discover that the CIF is always a convex function of its argument. Again, this conclusion is formally valid for $0 \leq \zeta \leq 1$. Note that the CIF for infinitely long signals, $\Im(\xi) \propto \sqrt{ } \xi$, has this property. An immediate consequence of convexity is that a signal of mean energy $N \bar{E}$ and duration $N^{\prime} \tau$ carries less information than $N N^{\prime}$ signals of energy $E$ and duration $\tau$.

To say more about the CIF we now interpret states like $a$ as (pure) quantum field states, and denote them by $|a>| b>,, \ldots$ with a priori probabilities $p_{a}, p_{b}, \ldots$ This is the full statistical description of our problem. Note the difference between this situation and the usual scenario in statistical mechnaics. There one uses the full density operator, and for consistency the von Neumann quantum formula for entropy. ${ }^{34}$ Here we only use the density operator's diagonal terms, namely the probabilities $\left\{p_{a}, p_{b}, \ldots\right\}$. The off-diagonal parts describe correlations which are foreign to the business at hand. Were we to include them in the description, we would get contributions to the information of signals which could not be ferreted out by a receiver whose job is to distinguish one pure state from another.

To make things simpler let us assume the signaling field is a free field. If it is subject to interactions (arguably it must be if communication is to be possible), we assume that our choice of propagating normal modes manages to eliminate any cross interaction terms, e.g. normal modes in an elastic solid. The field hamiltonian thus corresponds to a collection of noninteracting harmonic oscillators, one for each field mode. Depending on what it takes to do this, the quanta will be free particles, e.g. photons, or quasiparticles, e.g. phonons.

Consider a single mode $j$. To it corresponds a harmonic oscillator hamiltonian $H_{j}$ with a certain frequency $\omega_{j}$. One type of states of mode $j$ are the occupation number states $\mid j \alpha>$ defined by $H_{j}\left|j \alpha>=n_{\alpha} \hbar \omega_{j}\right| j \alpha>$ where $n_{\alpha}$ is a nonnegative integer. Other choices like coherent and squeezed states ${ }^{24}$ are not eigenstates of the mode hamiltonian. However, any such state $|j \beta\rangle$ does have a well defined mean energy $\varepsilon_{j \beta}$ :

$$
\varepsilon_{j \beta}=<j \beta\left|H_{j}\right| j \beta>\text {. }
$$

We can now build the signal states $\mid a>$ by exploiting the independence of the $H_{j}$, namely,

$$
|a>=| i \alpha>\otimes|j \beta>\otimes| k \gamma>\cdots
$$

where $i, j, k, \ldots$ label modes, $\alpha, \beta, \gamma \ldots$ label one-mode states, and $a, b, \ldots$ label signal (many-mode) states.

The probabilities $p_{a}$ of the signal states are assumed to be normalized to unity, but it is unnecessary for the signal states to form a complete set in the sense of quantum theory. However, completeness obviously favors higher communication rates by making a maximum number of states available, and will be assumed henceforth. We start by defining the mean energy of the signal:

$$
\bar{E}=\sum_{a} p_{a}\left(\varepsilon_{i \alpha}+\varepsilon_{j \beta}+\cdots\right) .
$$

Two averages are involved here: a quantum expectation value over the one-mode states which yields $\varepsilon_{i \alpha}+$ $\varepsilon_{j \beta}+\cdots$, and a statistical average over the a priori probabilities for the signal states, $p_{a}$. Clearly, only the latter are involved in the calculations of $I_{\max }$. Thus from our point of view the expression $\varepsilon_{i \alpha}+\varepsilon_{j \beta}+\cdots$, though formally a quantum expectation value, can be treated as a definite energy $E_{a}$.

Turn now to the partition function, $Z$. The sum over $a$ is equivalent to one over all combinations of $j$ and $\alpha$. Thus in a manner analogous to well known thermodynamic calculations, $Z$ can be written as $\prod_{j} Z_{j}$ where

$$
Z_{j}(\mu) \equiv \sum_{\beta} e^{-\mu \varepsilon_{j \beta}}=\sum_{\beta} \exp \left(-\mu<j \beta\left|H_{j}\right| j \beta>\right) .
$$

Contrary to naive expectations, the sum in Eq.(57) is not invariant under a unitary transformation of the $\mid j \beta>$ because the exponentiation process precedes the trace. This means that the channel capacity may vary 
with the type of quantum states $\mid j \beta>$ used. In the next two sections we study communication via occupation number states. They are contrasted with coherent states in Sec.3.5. Sec.3.6. presents a theorem showing that occupation number states are indeed optimal ones (maximum communication rate for given energy), but are not unique in this respect.

\subsection{Occupation Number States}

Occupation number states are relevant, for example, for an optical fiber communication channel with a photoelectric tube equipped with photon counting electronics as a detector. Our full attention will here be given to the propagation of signals and we shall ignore questions involved in the reception. These last have been treated by Yamamoto and Haus ${ }^{24}$ and Saleh and Teich. ${ }^{26}$

If the states $\mid j \beta>$ are chosen as occupation number states, $<j \beta\left|H_{j}\right| j \beta>=n_{\beta} \hbar \omega_{j}$. For a bosonic field $n_{\beta}$ can be any nonegative integer. Thus for bosons $Z_{j}$ reduces to the partition function of a harmonic oscillator at temperature $\mu^{-1}$ :

$$
Z_{j}=\sum_{n=0}^{\infty} e^{-\mu n \hbar \omega_{j}}=\left[1-e^{-\mu \hbar \omega_{j}}\right]^{-1}
$$

The calculation for fermions is quite similar. ${ }^{15}$ To calculate $Z$ we first sum $\ln Z_{j}$ over modes, and then exponentiate the result. In general the sum has to be done numerically. However, in the small $\mu$ limit we can perform the sum analytically in the continuum approximation.

For small $\mu$ the exponent in Eq.(58) changes gradually with $\omega_{j}$ so that we may replace the sum over $Z_{j}$ by an integral according to the usual rule $\sum_{j} \rightarrow \tau \int d \omega / 2 \pi$. The integral is a familiar one from the statistical mechanics of a one-dimensional Bose gas, and the final result is

$$
Z(\mu)=\exp \left(\frac{\pi \tau}{12 \mu \hbar}\right)
$$

Our brief derivation here glosses over the question of dispersion (signal speed depending on frequency). It can be shown ${ }^{15}$ that all effects of dispersion cancel out if the various modes are properly sequenced.

Clearly for small $\mu$ (more precisely small $\mu \hbar \tau^{-1}$ ), $Z \gg \zeta$ so that $C \approx Z^{-1}$ for self-heralding signals. For heralded signals this is, of course, an exact result. Eq.(52) now gives

$$
E=\pi \tau / 12 \hbar \mu^{2} .
$$

Calculating $I_{\max }$ from Eq.(53) and eliminating $\mu$ between the results gives in the continuum limit

$$
I_{\max } \rightarrow(\pi E \tau / 3 \hbar)^{1 / 2} \log _{2} e \text { bits. }
$$

Apart from the numerical constant this is just Marko's ${ }^{6}$ expression for $I_{\max }$. It reduces to the Pendry formula (16) under the substitutions $E / \tau \rightarrow P$ and $I_{\max } / \tau \rightarrow \dot{I}_{\max }$. Thus as anticipated, for large $\xi \Im(\xi) \rightarrow$ const. $\sqrt{ } \xi$ and the difference between heralded and self-heralding signals disappears. Comparing Eq.(51) and (59) we see that the differences between heralded and self-heralding cases disappear when $\tau / \mu \hbar \gg 10$. From (60) we see that the merging should be apparent for $\xi \gtrsim 10^{2}$, which can also be taken as the criterion for approach to the limit (61). Thus the long duration signals for which the results of Sec.2.4. apply are those with $E \tau / \hbar \gtrsim 10^{2}$.

For $\xi<10^{2}$ the continuum approximation is inappropriate and we must go into some detail regarding the form of the spectrum $\left\{\omega_{j}\right\}$. The burst signal as seen from a fixed point may be represented by some function $F(t)$ which has compact support in time i.e., it is nonvanishing only in the interval $[0, \tau]$. In fact, it is mathematically convenient to regard $F$ as periodic with period $\tau$. This "periodic boundary condition", well known from quantum physics, captures the essence of the finiteness of the duration while keeping the mathematics simple. Resolve $F(t)$ into its Fourier components involving the angular frequencies $2 \pi j \tau^{-1}$ for all positive integers $j$ (negative integers are superfluous - recall that under second quantization of a Bose field negative frequencies just duplicate the modes). The $j=0$ (dc) mode may be be ignored; it can be argued (see Sec.5.4.) that it corresponds to a condensate of the field to which no entropy (information) can be ascribed. So the spectrum is $\omega_{j}=2 \pi j \tau^{-1}$ with $j=1,2 \ldots$, and with no degeneracies.

Using (58) we now write the partition function (50) as

$$
\ln Z=-\sum_{j=1}^{\infty} \ln \left(1-e^{-b j}\right)
$$


and the mean energy (52) as

$$
\bar{E}=\frac{2 \pi \hbar}{\tau}\left(\frac{Z}{Z-\zeta}\right) \sum_{j=1}^{\infty} \frac{j}{e^{b j}-1},
$$

where $b \equiv 2 \pi \mu \hbar \tau^{-1}$. The parameter $b$ is to be chosen so that the desired value of $\bar{E} \tau / \hbar$ is reproduced by Eq.(63).

The continuum approximation (long duration signal) is accurate in the limit $b \rightarrow 0$. To deal with the case when $b$ is not small (brief signal), we carry out in Appendix B the sums in Eqs.(62)-(63) by means of the Euler-Maclaurin summation formula to obtain an approximation that transcends the validity of the continuum approximation. The results are

$$
\bar{E} \approx \frac{2 \pi \hbar}{\tau}\left(\frac{Z-\zeta}{Z}\right)\left[\frac{\pi^{2}}{6 b^{2}}-\frac{1}{2 b}+\frac{1}{24}\right]
$$

and

$$
\ln Z \approx \frac{\pi^{2}}{6 b}+\frac{1}{2} \ln b-\frac{b}{24}-0.91894 .
$$

We have checked numerically that Eqs.(64)-(65) are very accurate representations of (62)-(63) for $b<1$, and even at $b \sim 4$ their accuracy is better than 1\%; however, the accuracy deteriorates rapidly for larger $b$. At any rate, expression (64) for $\bar{E}$ is strictly positive as it should be.

Fig.1. The characteristic information function for occupation number states in the periodic boundary condition approximation as calculated from Eqs.(50)-(53).

The leading term in (64) and (65), which dominates for small $b$, corresponds precisely to Eqs.(59)-(61). It thus reproduces the results of the continuum approximation, and gives back the Pendry formula (16). In the general case Eqs.(64)-(65) together with Eqs.(51)-(53) give the CIF in parametric form. How does it look when $b$ is not small? Considering $\tau$ as fixed, let us first look at the case of heralded signals $(\zeta=0)$. This simplifies Eqs.(51), (53) and (63) considerably so that we get

$$
I_{\max }=\left(\frac{\pi^{2}}{3 b}+\frac{1}{2} \ln b-1.41894\right) \log _{2} e \text { bits. }
$$

Solving Eq.(64) for $b$ in terms of $\xi=E \tau / \hbar$ and susbtituting in (66) we get the form of the CIF:

$$
\Im(\xi)=\left(R \log _{2} e-\frac{1}{2} \log _{2} R-1.18808\right) \text { bits }
$$


with

$$
R \equiv 1+\left(1-\frac{\pi^{2}}{36}+\frac{\pi \xi}{3}\right)^{1 / 2}
$$

Since Eqs.(64)-(65) are accurate for $b<4$, we can use Eq.(67) for $\xi=\bar{E} \tau / \hbar>0.12$. As expected, $\Im \rightarrow \sqrt{ } \xi$ for large $\xi$ in which limit we recover Pendry's formula.

For self-heralding signals the factor $(Z-1) / Z$ in Eq.(64) cannot be ignored unless $Z$ is so large that the continuum approximation is acceptable. Hence, to get a full picture of the CIF in this case, as well as for heralded signals with small $\xi$, it is best to go back to the numerical evaluation of Eqs.(62)-(63). In Fig.1 we plot $I_{\max }$ vs. $\bar{E} \tau / \hbar$ on a $\log -\log$ scale. The dotted line is the CIF in the periodic boundary condition approximation for self-heralding signals while the dashed line refers to heralded signals. The solid line is the limiting formula (61) which is seen to be a strict upper bound on $I_{\max }$, and an excelent approximation to the CIF for signals with $\xi>10^{3}$ (these correspond to $I_{\max }>50$ bits). As $\xi$ decreases, $I_{\max }$ of finite duration signals falls below the naive prediction of (61) by a factor which reaches 2.5 for self-heralding signals with $\xi=10$. The corresponding true $I_{\max }$ is $\approx 2$ bits. Thus signals carrying modest information must be treated as finite duration signals, rather than by the steady state communication capacity.

Fig.2 displays the energy cost per bit $\epsilon_{\min }$ as a function of $I_{\max }$ in the self-heralded (dotted line) and heralded (dashed line) cases. The solid line corresponds once again to the limiting formula (61). Clearly for finite duration signals, the energy cost per bit exceeds that implied by the theory of steady-state communication. It may be seen that for self-heralding signals there exists a lower bound on the energy cost per bit of $\epsilon_{\min } \approx 4.39 \hbar \tau^{-1}$ which is attained for $I_{\max } \approx 3.5$ bits. No such bound exists for heralded signals: the energy cost per bit can be low for heralded signals with only fractions of a bit. Such low information signals are meaningful. For example, if a question has three alternative answers with the first being $98 \%$ probable, then 0.3 bits suffice to single out the answer [see Eq.(1)].

Fig.2. The energy cost per bit calculated under the conditions of Fig.1.

The periodic boundary condition assumes the modes in the signal have sharp frequencies. In truth if the signal has finite duration they should contain a continuum of frequencies. In Ref.15 Gabor's method of time-frequency cells ${ }^{35}$ has been used to justify the results obtained with the periodic boundary condition.

\subsection{Coherent States}

Coherent states can also be used for communication. In fact, in some sense they were the first to be used: a radio trasmitter produces an approximation to a coherent state of the electromagnetic field. In quantum optics the use of coherent and the closely related squeezed states in communication has been the subject of 
much interest. ${ }^{24-26}$ Let us investigate the maximum information that may be coded with coherent states. ${ }^{24}$ To avoid certain technical problems we concentrate on heralded signals $(\zeta=0)$.

A coherent state $^{36} \mid a>$ is defined as the tensor product a la Eq.(55) of eigenstates of the anhilation operators $\hat{a}_{j}$ of all the $N$ modes involved; thus

$$
\hat{a}_{j}\left|a>=\alpha_{j}\right| a>; \quad j=1,2, \ldots N
$$

As is well known, a coherent state can be expanded in occupation number states. ${ }^{36}$ In our case

$$
\left|a>=\prod_{j}^{N} e^{-\frac{1}{2}\left|\alpha_{j}\right|^{2}} \sum_{n_{j}} \frac{\alpha_{j}^{n}}{\sqrt{n_{j} !}}\right| n_{j}>.
$$

The energy $E_{a}$ associated with $\mid a>$ is the mean value of the Hamiltonian in $\mid a>$ :

$$
E_{a}=\hbar \sum_{j}^{N} \omega_{j}\left|\alpha_{j}\right|^{2}
$$

To calculate the information we shall need the partition function defined in (50). In view of (57), it may be written as $^{37}$

$$
Z(\mu)=\prod_{j}^{N} \int_{-\infty}^{\infty} \frac{d \alpha_{1} d \alpha_{2}}{\pi} e^{-\mu \hbar \omega_{j}\left(\alpha_{1}^{2}+\alpha_{2}^{2}\right)}
$$

where $\alpha_{j 1}$ and $\alpha_{j 2}$ are the real and imaginary parts of $\alpha_{j}$ and we have adopted the customary measure in $\alpha_{1}-\alpha_{2}$ space. ${ }^{36}$ Doing the Gaussian integrals gives

$$
Z(\mu)=\prod_{j}^{N} \frac{1}{\mu \hbar \omega_{j}}
$$

As before the lagrange multplier $\mu$ is fixed in terms of the specified mean energy by

$$
\bar{E}=-\frac{\partial \ln Z}{\partial \mu}=\frac{N}{\mu}
$$

Now we use (53) to calculate the maximum information that can be stored in the system with mean excitation energy $\bar{E}$ by coding with coherent $\operatorname{states}^{37}$ :

$$
I_{\max }=N \log _{2} e+\sum_{j}^{N} \log _{2}\left(\frac{\bar{E}}{N \hbar \omega_{j}}\right) \text { bits. }
$$

We note that for fixed $N$ this information becomes formally negative when $\bar{E}$ is so small that the energy per mode becomes much smaller than $\hbar \omega_{j}$ for a substantial fraction of the modes. We interpret this problem as due to the overcompleteness of the set of coherent states. ${ }^{36}$ At any rate it does not seem to be a problem for larger $\bar{E}$.

It is interesting to compare this result with the maximum information codable using an equal number $N$ of occupation number states. The partition function is the product of the $Z_{j}$ given by (58). The mean energy,

$$
\bar{E}=\sum_{j}^{N} \frac{\hbar \omega_{j}}{e^{\mu \hbar \omega_{j}}-1},
$$

is to be viewed as determining $\mu$ in terms of $\bar{E}$. Substitution in (53) gives

$$
I_{\max }=\mu \bar{E} \log _{2} e-\sum_{j}^{N} \log _{2}\left(1-e^{-\mu \hbar \omega_{j}}\right) \quad \text { bits }
$$


which in conjunction with (76) provides a parametric prescription for $I_{\max }(\bar{E})$. Evidently this $I_{\max }(\bar{E})$ is quite different from the one for coherent states, Eq.(75). When all $N$ modes have very similar frequencies (narrowband channel) it is easy to solve (76) for $\mu$, and subtitution in (72) gives

$$
I_{\max }=\left[\sum_{j}^{N} \log _{2}\left(1+\frac{\bar{E}}{N \hbar \omega_{j}}\right)+\frac{\bar{E}}{\hbar \omega_{j}} \log _{2}\left(1+\frac{N \hbar \omega_{j}}{E}\right)\right] \quad \text { bits }
$$

which could actually have been guessed from (45). It is a simple exercise to show that this $I_{\text {max }}$ exceeds that for coherent states for any $N$ and $\bar{E}$.

\subsection{The Optimum States for Signaling}

The previous discussion raises the natural question of which set of states leads to the maximum information transmission, other things being equal. It has often been stated that occupation number states are the best. ${ }^{24,26}$. The argument in support of this is that the capacity (45) may be obtained by maximizing Shannon's entropy subject to normalization of probability and stipulated mean occupation number (or mean energy for a narrowband channel). However, in this maximization the probabilities are regarded as depending on occupation number. ${ }^{24}$ Were the states characterized by some other quantum number, it is not certain that the resulting distribution and maximal entropy would be the same. The problem here is essentially that stated at the end of Sec.3.3. We now state and prove a theorem ${ }^{37}$ that clarifies the situation: occupation number states are indeed one of the sets of states which optimize information transmission (or information storage for that matter).

Let us imagine the change in $I_{\max }$ [as defined by given by (50)-(53)] due to an arbitrary variation of the set of states $\mid a>$. Since $\mu$ is representation dependent, its variation must be included. Thus

$$
\delta I_{\max }=[\delta(\mu \bar{E})+\delta \ln (Z-\zeta)] \log _{2} e .
$$

However, according to (50)

$$
\delta Z=-\sum_{a}\left(E_{a} \delta \mu+\mu \delta E_{a}\right) e^{-\mu E_{a}}
$$

where

$$
\delta E_{\alpha}=<\delta a|H| a>+<a|H| \delta a>
$$

whereas by $(52)$

$$
\bar{E}=-\frac{1}{Z-\zeta} \frac{\partial Z}{\partial \mu} .
$$

We find after substitution of all these in (79) that $\delta \mu$ cancels out. Therefore, the condition for $I_{\max }$ to be an extremum is

$$
\left.\delta \sum_{a} e^{-\mu E_{a}}\right|_{\mu}=0 .
$$

The problem of extremizing $I_{\max }$ with respect to the class of states is thus equivalent to extremization of the partition function at fixed $\mu$. This result is reminiscent of the thermodynamic rule that an equilibrium state characterized by a maximum of entropy for given mean energy amounts to a minimum of the Helmholtz free energy $F$ at given temperature. Since the partition function is just $\exp (-F / k T)$, we see that a maximum of the partition function is involved. This analogy tells us that the extremum sought in (83) is really a maximum. We may now prove the following theorem.

Theorem 2. $I_{\max }$ is maximized when the set of signaling states $\{|a\rangle\}$ is chosen as a complete set of eigenstates of the Hamiltonian $\hat{H} \cdot{ }^{37}$

Proof. From the variation principle in quantum mechanics ${ }^{36} E_{a} \equiv<a|\hat{H}| a>$ is extremal when $\mid a>$ is an eigenstate of $\hat{H}$. And if we insist that $<a|\hat{H}| a>$ be extremal with respect to a complete set $\{\mid a>\}$, then this is the set of (orthonormal) eigenstates of $\hat{H}$. Therefore, choosing $\{\mid a>\}$ as the set of eigenstates of $\hat{H}$ makes the partition function in (83) extremal with respect to small variations of the $|a\rangle$, thus satisfying (83). In fact, the partition function is maximized by this procedure. For according to the variational principle, the ground eigenstate gives the lowest possible $E_{a}$, the ground state eigenvalue. The next $E_{a}$ is the minimum possible within the set of states orthogonal to the ground state, and so on. It is clear that this set of minimum 
$E_{a}$ 's gives maximum $Z$. Therefore, by using a complete set of eigenstates of $\hat{H}$ as signaling states, we get maximum $I_{\max } \cdot \square$

We should mention that with the is choice of signaling states, the partition function $\mathrm{Eq},(50)$ is formally identical to the partition function from statistical mechanics, $\operatorname{Tr}(\exp (-\hat{H} / k T))$ as is clear by using the energy representation. Occupation number states of a free field are a special case of eigenstates of $\hat{H}$. Therefore, in the communication systems under consideration, channel capacity is maximized by using occupation number states (and measuring occupation number at the receiver).

\section{The Linear Bound on Communication}

As the example in Sec.3.4 shows, the CIF of a channel contains quite a lot of detail about the channel's communication capabilities; by the same token its computaion is quite an elaborate task. Sometimes the CIF description of communication is an expensive luxury. We might prefer a less detailed statement about the capacity which is easier to come by. It is under circumstances like these that the linear bound introduced by Bremermann ${ }^{9-11}$ is important. According to Bremermann, one can set a universal bound on channel capacity depending only on the signal's energy. His argument is that a signal, when looked at in quantum terms, must contain at least one quantum of some sort (in the language of Sec.3. it must be self-heralding). Thus for alloted signal energy $E$, the angular frequencies that can appear are bounded from above by $E / \hbar$. Bremermann interprets this as the bandwidth $\Delta \omega$ of the system relevant in the Shannon capacity formula (5). Regarding the signal-to-noise factor in the formula, Bremermann uses an obscure argument whose crux is to equate Shannon's noise with the energy uncertainty $\delta E \geq \hbar / \tau$ required by the time-energy uncertainty relation for a signal of duration $\tau$. His final result is ${ }^{11}$

$$
\dot{I}_{\max } \leq \frac{E}{2 \pi \hbar} \log _{2}(1+4 \pi) \text { bits s}{ }^{-1} .
$$

This bound involves no details of the channel's construction. Bremermann's argument has been critized ${ }^{8,14,15}$ for relying on the classical Shannon formula to get an ostensibly quantum result, and for the obscurity surrounding the connection of noise power with the time-energy uncertainty relation, itself a principle that invites confusion. ${ }^{38,39}$

However, there are other roads to the linear bound. For example, an independent argument ${ }^{12}$ for a bound like (84) relies on causality considerations combined with the bound on the entropy $H$ that may be contained by a physical system of proper energy $E$ and circumscribing radius $R$, namely,

$$
H \leq \frac{2 \pi E R}{\hbar c} .
$$

Originally inferred from black hole thermodynamics, ${ }^{16,19}$ this bound has since been established by detailed numerical experiments ${ }^{13}$ and analytic arguments ${ }^{13,20-23}$. According to Shannon's information theory, the peak entropy $H_{\max }$ for a system limits the maximum information $I_{\max }$ that can be stored in it. Now by transporting a system with information inscribed in it, one has a form of communication. Because the system cannot travel faster than light, it sweeps by a given point in time $\tau \geq R / c$ (issues connected with the Lorentz-Fitzgerald contraction are carefully dealt with in Ref.12.). Thus an appriate "receiver" can acquire from it information at a rate not exceeding $\tau^{-1} H_{\max } \log _{2} e$ (as usual, $\log _{2} e$ converts from nits to bits). Substituting from Eq.(85) we have

$$
\dot{I}_{\max } \leq \frac{2 \pi E}{\hbar} \log _{2} e \text { bits } \mathrm{s}^{-1}
$$

which is quite similar to (84). The drawback of this "derivation" is that it deals only with a very special sort of communication: information transfer by bulk transport. ${ }^{14}$

\subsection{Heuristic Derivation}

We now offer a heuristic argument ${ }^{40}$ for a communication bound of the form (86) which does not rely on the entropy bound Eq.(85). Suppose the information we wish to transmit is inscribed in a bosonic carrying field by populating its energy levels with quanta; each quantum configuration represents a different symbol. Let $\tau$ and $E$ be the signal's duration and energy, respectively, $\epsilon$ the lowest non-zero one-quantum energy level, and $\Delta \epsilon$ the smallest energy separation between levels beneath $E$. Evidently the total number of occupied 
levels is $N \leq \frac{E}{\Delta \epsilon}$, while the total number of quanta is $M \leq E / \epsilon$. The total number of configurations $\Omega$ must thus bounded from above by the number of configurations of a system composed of $M=E / \epsilon$ identical bosons distributed among $N=\frac{E}{\Delta \epsilon}$ levels. This last is given by a formula well known from Bose statistics. Therefore,

$$
\Omega \leq \frac{(N+M-1) !}{M !(N-1) !}
$$

All these configurations are a priori equally likely so that the peak entropy of the signal is bounded according to

$$
H_{\max } \leq \ln [(N+M-1) !]-\ln M !-\ln [(N-1) !] .
$$

Assuming $N$ and $M$ are large, the logarithms may be approximated by Stirling's formula. Substituting the bounds on $N$ and $M$, equating $H_{\max }$ with the peak information, and converting to bits we get

$$
I_{\max } \leq \frac{E}{\sqrt{\epsilon \Delta \epsilon}}\left[\left(\frac{\epsilon}{\Delta \epsilon}\right)^{1 / 2} \log _{2}\left(1+\frac{\Delta \epsilon}{\epsilon}\right)+\left(\frac{\Delta \epsilon}{\epsilon}\right)^{1 / 2} \log _{2}\left(1+\frac{\epsilon}{\Delta \epsilon}\right)\right] \text { bits. }
$$

The function $f(x)=x^{-1 / 2} \log _{2}(1+x)$ appearing here is already familiar from Sec.2.3. It follows from its properties that the term in square brackets in Eq.(89) can be no larger than $\approx 2.32$. Now in order to be able to decode the information, the receiver must be able to distinguish between the various enrgy levels, which calls for energy measurement with precision $\delta E \leq \Delta \epsilon$. According to the time-energy uncertainty principle, the finiteness of the measurement interval $\tau$ imposes an uncertainty $\delta E \geq h / \tau$. Thus, $\Delta \epsilon \geq h / \tau$ in order that the useful information approach $I_{\max }$. Furthermore, if $R$ is the spatial extent of the signal, we can use the momentum-position uncertainty relation to set the bound $\epsilon / c \geq \hbar / R$. In addition, on grounds of causality the inequality $\tau \geq R / c$ must apply. Therefore, $\sqrt{\epsilon \Delta \epsilon} \geq \sqrt{2 \pi} \hbar \tau^{-1}$. It follows from (89) that

$$
\dot{I}_{\max } \leq \frac{0.925 E}{\hbar} \text { bits s}^{-1}
$$

which is of the same form as (86).

This argument, appealing as it is, suffers from two drawbacks: it is only valid for large $\mathrm{N}$ and $\mathrm{M}$, where Stirling's approximation may be trusted, and it makes use of the popular but nonrigorous version of the time-energy uncertainty relation. We now turn to two exact derivations of the linear bound.

\subsection{The Bound for Signals with Prescribed Mean Energy}

The discussion here will refer to self-heralding signals only. We assume the signal states $|a\rangle$ to be occupation number states specified by the list of occupation numbers $\left\{\mathrm{n}_{1}, \mathrm{n}_{2}, \ldots\right\}$ for the various modes. Each state $|a\rangle$ is ascribed a particular a priori probability $p_{a}$ subject to the normalization condition $\sum_{a} p_{a}=1$. The information that may be carried by the signal is limited by Shannon's entropy (1). The energy of the state $\mid a>$ is evidently $\sum_{j} n_{j} \epsilon_{j}$ with $\epsilon_{j}=\hbar \omega_{j}$. The signal's mean energy is

$$
\bar{E}=\sum_{a} p_{a} \sum_{j} n_{j} \epsilon_{j}
$$

We maximize $H / \bar{E}$ subject to (91) by means of the variational principle

$$
\delta\left(-\frac{\sum_{a} p_{a} \ln p_{a}}{\sum_{a} p_{a} \sum_{j} n_{j} \epsilon_{j}}-\lambda \sum_{a} p_{a}\right)=0,
$$

where $\lambda$ is a Lagrange multiplier. Notice that, in contrast with similar calculations in statistical mechanics, one is not here enforcing an energy constraint. Calling $(H / E)_{\max } \equiv \mu$, variation of $p_{\alpha}$ gives

$$
\left(\ln p_{a}+\mu \sum_{j} n_{j} \epsilon_{j}+\lambda \bar{E}\right) \delta p_{a}=0
$$

Thus for nonvacuum states the probability distribution reads,

$$
p_{a}=C e^{-\mu \sum_{j} n_{j} \epsilon_{j}}
$$


where $C$ is a normalization constant into which we have absorbed the Lagrange multiplier $\lambda$. Of course, when all $n_{j}=0$, the probability is taken to vanish (self-heralding signal).

When we compute the Shannon information from (53) with the maximizing distribution (94) we get

$$
I_{\max }=\mu \bar{E}-\ln C .
$$

However, $\mu$ is defined as the maximal $H / \bar{E}$, and since $\bar{E}$ in (93) refers to the mean energy of the maximal $H / \bar{E}$ situation, it follows that $C=1$ for consistency. Thus despite the similarity between our distribution and Boltzmann's, the "inverse temperature" $\mu$ here is not a free parameter, but is fixed by the normalization of probability. Now according to (49) and (51) for self-heralding signals, the constraint $C=1$ implies that $Z=2$. Writing $Z=\prod_{j} Z_{j}$ and using the form (58) we find that $\mu$ is a root of the equation

$$
-\sum_{j} \ln \left(1-e^{-\mu \epsilon_{j}}\right)=\ln 2
$$

Because each term in the sum increases with $\mu$, it follows that the root is unique.

Let us now use the periodic boundary condition approximation according to which $\epsilon_{j}=2 \pi j \hbar \tau^{-1}$ with $j=1,2, \ldots \quad$ Numerical summation gives that $\mu \pi \hbar \tau^{-1} \approx 0.4931$. Since $\mu$ is the maximum of $H / E$, it follows after conversion to bits that

$$
\dot{I}_{\max } \leq 0.2279 \mathrm{E} / \hbar \text { bits s}^{-1},
$$

which is of the same form as the heuristic bound (90), but tighter.

The equality in (97) would corresponds to a line of slope unity in Fig.1. just tangent to the self-heralding curve at its knee. It may be seen that for moderate signal information $(<10$ bits $)$, the linear bound gives a considerably better idea of the CIF than does the Pendry formula. The opposite is true for large information. The linear bound may be saturated only for signals which carry about 3 bits. The point that maximum communication rate is attainable only for a signal carrying of the order of one bit was made early by Landauer and Woo. ${ }^{41}$

Had we worked with heralded signals we would have found that there is no maximal $H / E$ for given $\tau$. This is already clear from Fig.1. Does this weaken the generality of the claimed linear bound? In this respect it is important to notice that the violation shows up only for very low signal mean energy. This means that only the lower energy levels are populated, and sparsely at that. Now in Bose statistics of one level, the ratio of mean energy $E$ to energy standard deviation $\Delta E$ is $N^{1 / 2}$ where $N$ is the total number of quanta. Thus when the violation of the linear bound appears, the system has few quanta and the energy spread is not small compared to the mean energy itself. Hence mean energy is far from representing the actual energy. The conclusion must be that the characterization of a signal by mean energy is not appropriate in that regime where the linear bound seems to break down. We can deal with this problem as follows.

\subsection{The Bound for Signals with Specified Energy Budget}

Instead of specifying the signal by its mean energy, a misleading concept for low excitations, one can instead specify the energy "budget" or energy "ceiling" for signaling - the maximum available energy per signal. Shannon's entropy Eq.(1) reduces in this case to Eq.(2) since all signal states with energies below the maximum are equally likely. The problem reduces to counting the number of signal states as a function of the energy budget. This is a difficult problem in general, as has long been known from its analog in microcanonical statistical mechanics. This counting was carried out numerically for a few examples relevant to communication by Gibbons, ${ }^{42}$ and later by one of us. ${ }^{13}$ Recently progress has been made towards the limited goal of establishing analytically bounds in the number of quantum states up to a given ceiling energy for three dimensional systems. ${ }^{23}$ Here we review a one dimensional version of these results which applies to signals of finite duration. ${ }^{40}$ In Sec.5. we shall use the more general result ${ }^{23}$ to discuss limitations on information storage.

Let $\Omega(E)$ be the number of distinct quantum states of a system accessible with energy not exceeding $E$. Evidently $\Omega(E)$ depends on the one-quantum energy spectrum $\left\{\epsilon_{j}\right\}$. According to Eq.(2) the maximum (microcanonical) information that may be coded in the system corresponds to the entropy

$$
H_{\max }=\ln \Omega(E) .
$$


Now focus attention on configurations with a fixed number of indistinguishable quanta $m$. If the oneparticle levels are ordered by energy, so that $\epsilon_{j_{1}} \leq \epsilon_{j_{2}}$ when $j_{1}<j_{2}$ (degenerate levels are to be ordered arbitrarily), an $m$-quanta configuration is specified by the set of occupied one-quantum levels $\left\{\epsilon_{j_{1}}, \epsilon_{j_{2}}, \ldots\right\}$ (of course, some of them may be repeated, corresponding to multiple occupation of a level). The number $\Omega_{m}(E)$ of $m$-quanta states with total energy $\leq E$ can be written as

$$
\Omega_{m}(E) \equiv \sum_{j_{1} \leq j_{2} \leq \ldots j_{m}} \Theta\left[E-\epsilon_{j_{1}}-\epsilon_{j_{2}} \ldots-\epsilon_{j_{m}}\right] \quad m \geq 1,
$$

where $\Theta$ is Heavyside's function. The disposition of the limits on the summation has the effect of avoiding double counting of states which differ only by the exchange of (identical) quanta. We shall assume a nondegenerate vacuum so that $\Omega_{0}(E)=1$ for $E \geq 0$. The number of one-quantum states with energy up to $E$,

$$
n(E) \equiv \Omega_{1}(E)=\sum_{a=0}^{\infty} \Theta\left(E-\epsilon_{j}\right),
$$

will play a key role in further discussion in Sec.5. We assume there is no zero-mode i.e., $\epsilon_{j}>0$. Thus $n(E)=0$ for $E \leq 0$.

The problem of finding the number of accessible quantum states, $\Omega(E)$, can evidently be reduced to that of counting all possible $m$-quanta states:

$$
\Omega(E)=\sum_{m=0}^{\infty} \Omega_{m}(E)
$$

Explicit calculation of $\Omega(E)$ by this means is not, in general, practical. However, bounds can be set on it by the following procedure. Relaxing the energy ordering in Eq.(99), we define the useful auxiliary quantity $N_{m}(E)$ which overcounts the number of $m$-indistinguishable quanta configurations $\left[\Omega_{m}(E)<N_{m}(E)\right]$,

$$
N_{m}(E) \equiv \sum_{j_{1}, j_{2}, \ldots} \Theta\left[E-\epsilon_{j_{1}}-\epsilon_{j_{2}} \ldots-\epsilon_{j_{m}}\right] \quad m \geq 1
$$

and

$$
N_{0}(E) \equiv \Theta(E)
$$

In analogy with equation (101), it is natural to define

$$
N(E) \equiv \sum_{m} N_{m}(E)
$$

which overcounts the number of accessible quantum states:

$$
\Omega(E)<N(E) .
$$

The advantage of this procedure is that $N(E)$ satisfies a very simple integral equation (see Appendix $\mathrm{C}$ ):

$$
N(E)=\Theta(E)+\int_{0}^{E} N\left(E-E^{\prime}\right)\left(\frac{d n}{d E^{\prime}}\right) d E^{\prime} .
$$

Let us take the Laplace transform of this equation. Denoting the Laplace transform of a function $f(E)$ by $\widetilde{f}(s)$, and making use of the convolution theorem, and of the fact that $n(0+)=0$, we obtain

$$
\widetilde{N}(s)=\frac{1}{s[1-s \widetilde{n}(s)]} .
$$

This equation plays a central role in our discusion.

As we saw in Sec.4.2., in the periodic boundary condition approximation for a signal with period $\tau$, the one-quantum spectrum is $\epsilon_{j}=j \epsilon ; j=1,2, \ldots$, where $\epsilon=2 \pi \hbar \tau^{-1}$. Thus, the Laplace transform of the one-quantum particle number function is

$$
\widetilde{n}(s)=\int_{0}^{\infty} d E e^{-E s} \sum_{j=1}^{\infty} \Theta(E-j \epsilon)
$$


which can be cast in the form

$$
\widetilde{n}(s)=s^{-1} \sum_{j=1}^{\infty} e^{-j \epsilon s} .
$$

Performing the sum in (109), substituting in (107), and inverting the Laplace transform $\widetilde{N}(s)$ we have

$$
N(E)=\frac{1}{2 \pi i} \int_{\gamma-i \infty}^{\gamma+i \infty} \frac{e^{s \epsilon}-1}{s\left(e^{s \epsilon}-2\right)} e^{E s} d s
$$

where $\gamma$ must be chosen to the right of the poles located in the $s$-plane at $s=\epsilon^{-1}(\ln 2+i 2 \pi k), k=$ $\{\ldots,-2,-1,0,1,2, \ldots\}$. It should be noted that there is no pole at $s=0$.

This integral has been evaluated by the contour method ${ }^{40}$ and is reproduced in Appendix D; the result is

$$
N(E)=2^{[[E / \epsilon]]}
$$

where $[[x]]$ stands for the whole part of $x$. Thus whenever $0 \leq E / \epsilon \leq 1$ we get that $N(E)=1$ and $H(E)=0$. This confirms the feeling that as long as the first energy level is not accessible, no information can be encoded at all. Recalling the definition of $\epsilon$, the fact that $I_{\max } \leq \log _{2} N(E)$ and the convention $\dot{I}_{\max }=I_{\max } / \tau$, we get

$$
\dot{I}_{\max } \leq \frac{E}{2 \pi \hbar} \text { bits s}^{-1}
$$

which is consistent with Eqs.(86), (90) and (96).

\subsection{Caveats on the Derivation of the Linear Bound}

As it first appeared in Bremermann's work, the linear bound on capacity was predicated on the supposition that a signal must consist of at least one quantum. Our discussion of self-heralding vs. heralded functions in Sec.3.2. makes it clear that the vacuum state is a legal signal state under some circumstances, so that Bremermann's supposition is not generic. It would thus appear that the linear bound cannot be valid under all circumstances. Indeed, in Sec.4.2. we found that for signals with specified mean energy, the linear bound (whatever the exact numerical coefficient) can be surpassed by heralded signals (see especially Fig.1). It turns out, somewhat surprisingly, (Sec.4.3) that for signals with specified energy ceiling, the bound is valid regardless of whether the signal is heralded or not (the vacuum was included in the list of states). Thus, the linear bound turns out to be very general.

Three ingredients went into the proof of the linear bound in Sec.4.3.: the periodic boundary condition approximation, the assumption that the zero frequency mode cannot be used in signaling, and the characterization of signals by occupation number. Let us discuss them in turn.

By viewing the signal as periodic one obtains a simple form for the frequency spectrum. This sort of approach is quite common in physics. Arguably, it would have been more realistic to look at signals that turn on and off abruptly. In that case there are no sharp one-quantum energies; rather all levels are broadened. One way to proceed then is to use Gabor frequency-time cells ${ }^{35}$ to partition the phase space occupied by the signal. To each such cell is assigned a Gaussian modulated sinusoidal wave which takes over the role of the pure sinusoidals in the Fourier representation of the periodic signal, and embodies the idea that the energy levels must be broadened in inverse proportion to the duration $\tau$. If all cells are chosen to extend a time $\tau$, it is natural to choose the central frequencies of the Gaussian wavepackets to correspond to the energies $\epsilon_{j}=2 \pi j \hbar \tau^{-1}$, precisely the frequencies figuring in the periodic boundary condition approximation. ${ }^{15}$ The energy spread of a wavepacket is then $\sim 2 \pi \hbar \tau^{-1}$. With this choice it is easy to grasp the effect of the periodic boundary condition approximation.

For energy ceiling $E$, a many-quanta state with $\sum_{j} \epsilon_{j}>E$ was excluded in the periodic boundary condition approximation. However, if the energy sum exceeds $E$ only by a quantity of order $2 \pi \hbar \tau^{-1}$, the state is allowed in the present description because it is possible for the true energies of several of the quanta to be on the low side of the central energies of their Gaussian packets. Of course, the larger the excess of $\sum_{j} \epsilon_{j}$ over $E$, the less probable the state. For if the state is a one-quantum state, the quantum's energy must lie on the outskirts of the Gaussian packet to keep below $E$. This situation has low probability. If we deal with a several-quanta state, the individual energies can lie closer to the central energies, but there must be a trend toward the lower energy side. Thus, although the individual quanta are not at very improbable 
energies, the product of several probabilities smaller than one will cause the overall configuration to be unlikely. Thus in the exact treatment extra states become available, but these states have low probability.

We must also note that some states which were permitted in the periodic boundary condition approximation become, in the exact treatment, low probability states. These are states with $\sum_{j} \epsilon_{j}$ within a quantity $\lesssim 2 \pi \hbar \tau^{-1}$ on the low side of $E$. This is because with nonnegligible probability some of the quanta involved can lie on the high side of their Gausssian peaks, and cause the true total energy to exceed $E$. This effect partly neutralizes the gain of states discussed above. The conclusion must be that the periodic boundary condition approximation is likely to only somewhat underestimate the number of states. We thus venture to conclude that (112) is likely to be only a little below the true linear bound in the exact treatment.

In our derivation of the linear bound in Sec.4.3. we excluded the mode with $\epsilon_{j}=0$. If included it would have led to an infinity of states for any energy. This is because we can form arbitrarily many states by having a varying number of quanta with zero energy; all these are permitted being below the energy ceiling. To understand why the zero frequency mode must be excluded, one must distinguish between the situation where the signal is periodic, and the one where it is sharply limited in time. In the first case the periodic boundary condition is exact; the zero frequency mode in question sets the dc level of the signal. This dc level cannot serve to send information. It is permanent, and does not turn on when the signal is sent so that the signal's information is not coded in it. At best the dc level conveys some information about the channel, but not specific to the signal. The zero frequency mode thus has no role in signaling. When the signal is sharply bounded temporally, the spreading of frequencies precludes the existence of a mode with exactly zero energy. Even if interpreted as the center of a Gaussian wavepacket, $\epsilon_{j}$ cannot vanish: that would entail negative as well as positive frequencies. Hence in the periodic boundary condition approximation, we should exclude the $\epsilon_{j}=0$ mode.

In our derivation the signal states were classified by occupation number. As shown in Sec.3.6., these maximize the capacity. Therefore, the bound on capacity (112) must apply to all kinds of signal states.

\subsection{The Linear Bound and the Time-Energy Uncertainty Relation}

What is the linear bound good for? First of all, it serves as a convenient rule of thumb for estimating peak performance possible for a communication system based on one channel and signals of finite duration. Indeed, very often one may not want to delve into details of the system which would be necessary to determine the full CIF. At the risk of extreme generosity we can then use the linear bound to estimate the capacity. For example, if a budget of $1 \mathrm{ev}$ is available per signal, we estimate from (112) that $\dot{I}_{\max }<3.8 \times 10^{13} \mathrm{bits} \mathrm{s}^{-1}$. Certainly no optical channel has been known to exceed this bound.

Another application of the linear bound is a reinterpretation of the time-energy uncertainty relation. The canonical statement of the time-energy uncertainty relation is ${ }^{38}$ : the product of the dispersion in the energy of a system and the timescale over which the expectation value of a system observable varies is $\geq \hbar / 2$. However, the "popular" version of the time-energy uncertainty relation has it that: the energy (or the dispersion in the energy) of a system times the duration over which it is measured $\gtrsim h$ (here $h$ is the quantum of action $2 \pi \hbar$ ). The popular version is not a theorem, but it may be employed pragmatically (at ones risk) in a case by case basis. ${ }^{39}$ Indeed, some studies of quantum capacity ${ }^{5,10,11}$ (and even one of our own arguments in Sec.4.1.) make use of it. Sometimes it is misleading. For example, it would seem to forbid signals with finite $\tau$ and arbitrarily small $E$; yet such (heralded) signals are possible and meaningful, as mentioned in Sec.3.4.

With help of our result (112), we may reinterpret the time-energy uncertainty relation as follows. The energy cost per bit in communication, defined as $E / I_{\max }$, has been mentioned often. Suppose we carry out a measurement, obtaining thereby some information, which is then conveyed to the observer by a communication channel. The product of the energy cost per bit of the signal and the time interval $\tau$ during which the information is delivered is no smaller than the quantum of action $h$. This follows from (112). This statement is quite different from the canonical statement of the time-energy uncertainty relation quoted earlier. We believe it is more useful in the context of quantum measurement because it refers to energy of the signal, not to energy dispersion of the sytem, and it talks of the time interval over which the information is delivered, not merely of a timescale of the system.

\subsection{The Linear Bound for Many Channels}

Up to now we have dealt only with communication via a single channel. Communication systems are 
more often than not multichannel ones, e.g. waveguide with two polarizations, television, fiber optics link, optical nerve, ... How does the capacity of a multichannel system compare with that of a single one? More specifically, how are formulae like the Pendry bound (16), or the linear bound, to be adapted to the manychannel case? Both Pendry ${ }^{8}$ and Levitin ${ }^{43}$ have rightly stressed the difficulty in formulating a universal bound on $\dot{I}$ for information flow in three dimensions (many spatial channels). A similar point was made earlier by Landauer and $W_{0 o}{ }^{41}$. By contrast Bremermann ${ }^{11}$ regarded his bound, Eq.(84), as also valid in the multichannel case. Rather than review the entire issue of multichannel communication, let us clarify this controversy by analyzing the linear bound in the context of an array of parallel channels which partake of the total mean energy $\bar{E}$.

We assume the signal velocity $c_{s}$ is constant and identical for all channels. This makes the task of synchronizing the arrival of signals via the diverse channels rather straightforward. Such synchronization is evidently a prerequisite for maximizing the communication rate (staggered signals stretch the duration of the reception). Since the signal with the longest duration sets the characteristic time $\tau$ out of which $\dot{I}$ is computed, one can always make the overall $\dot{I}$ larger by rearranging the information among the various channels so that the durations of the signal are similar in all of them. Therefore, we shall assume that the durations of the signals in the various channels are the same.

We shall study the linear bound only for self-heralding signals by the method of Sec.4.2. The difference now is that a state $|a\rangle$ is here defined as a set of occupation numbers for the various modes $j$ in all the channels. We suppose there are $N$ channels which we label by Greek subscripts like $\nu$. We shall denote $\prod_{j}\left(1-e^{-\mu \epsilon_{j}}\right)^{-1}$ taken over channel $\nu$ by the symbol $Y_{\nu}$. First we shall consider what we shall call a simple communication system, one whose channel architecture is orderly enough that it suffices for the signal to exhibit one quantum in some channel in order for its arrival to be unambiguously noted, e.g. an optical fiber link where the fibers are not twisted or otherwise jumbled,... The probabilities $p_{a}$ for all states are still given by Eq.(94), except that the vacuum in all channels is assigned vanishing probability. Therefore, the normalization of probability gives

$$
C \prod_{\nu=1}^{N} Y_{\nu}-C=1,
$$

where the last $C$ corrects for the inclusion of the vacuum in the product. As in Sec.4.2. we find that necessarily $C=1$ in order for the situation to correspond to maximum $H / I$. From (113) follows a condition on $\mu$ just like Eq.(96), but with the sum being also over channels.

If all $N$ channels are of the same sort, all contribute the same factor $Y_{\nu}$. This reflects the fact that maximum information transmission obtains when the signal energy is shared out equally among all channels. This is like endowing all channels with the same "temperature" $\mu^{-1}$ so that all the $Y$ 's are identical. In conclusion, the condition on $\mu$ is

$$
-N \sum_{j} \ln \left(1-e^{-\mu \epsilon_{j}}\right)=\ln 2
$$

where now the mode sum is over one channel. Since the mode sum (including the minus sign) is monotonically decreasing with $\mu$, it follows that when $N \gg 1,2 \pi \mu \hbar \tau^{-1} \gg 1$ (for $N=1,2 \pi \mu \hbar \tau^{-1}=0.986$ according to Sec.4.2). In this case the mode sum is dominated by its first term, which may be approximated by $\exp \left(-2 \pi \mu \hbar \tau^{-1}\right)$. We thus obtain a simple expression for $\mu$. Recalling that $\mu=(H / I)_{\max }$ and converting to bits we have

$$
\dot{I}_{\max } \leq \frac{E}{2 \pi \hbar} \log _{2}\left(\frac{N}{\ln 2}\right) ; \quad N \gg 1 .
$$

This bound should be compared with (97). The well known tendency for logarithmic growth of the capacity with the number of channels is evident here. The difficulty in stating a universal capacity or bound for multichannel communication ${ }^{8,41,43}$ is thus clear.

In the paradigm just considered, which is relevant for many man-made communication systems, the basic requirement is that only the state which is vacuum in all channels is to be excluded. Yet in many naturally occurring communication systems, whose channel "architecture" is complex or disorderly, this would be too weak a requirement. A case in point is provided by the bundle of electromagnetic channels through which an astronomer acquires information about a supernova explosion in a distant galaxy. The relevant channels are a set of photon channels whose propagation directions all fall in the tiny solid angle subtended in the sky by the galaxy. Before the outburst became visible, the astronomer did not know which of all the channels available to him are operative. As he becomes aware of the explosion, the operative set is fixed by the 
presence of photons appearing at random in some of a small subset of all channels that our astronomer was monitoring. A second example may be provided by the optical nerve (a bundle of electrochemical channels) which conveys information to the visual cortex in the brain from a vast number of optical receptors in the eye's retina. When under dim illumination an object becomes visible in part of the eye's field of view, the firing of neurons in a few randomly chosen fibers belonging to the subset of the nerve that monitors the relevant part of the field delimits which group of channels is operative for the particular sighting.

Abstracting from our examples, we define a spatially blurred communication system as one in which the operative set of channels is determined when a signal is received by the presence of at least one quantum in each of at least a fraction $r$ of the channels, with the populated channels being selected at random. We call $r$ the filling fraction of the communication system. Determination of $r$ must depend on details of the physics and required reliability of the communication system. Here we shall only be concerned with the dependence of $\dot{I}_{\max }$ and $\epsilon_{\min }$ on the assumed filling fraction.

To formulate the theory of blurred communication systems, we imagine a system with a large number $N$ of channels. Allowed signals must have at least one quantum in each of $M$ channels chosen at random out of the $N$. The value $M$ is chosen so that $M / N$ approximates $r$ as $N$ and $M$ are made large. This realization of the system will be justified if the ultimate results depend only on the ratio of $M / N$, and not on $N$ and $M$ separately. Recalling the development in Sec.4.2., we see that the normalization condition for the $p_{a}$, which determines $\mu$, can be put in the form (recall $C=1$ )

$$
\prod_{\mu=1}^{M} Y_{\nu}-\sum_{\nu>\rho>\cdots} Y_{\nu} Y_{\rho} \cdots=1
$$

where each term in the sum has $M-1$ distinct factors. The first term in Eq.(116) is, appart from the factor $C$, the total probability of all conceivable states. From it is deducted the formal [according to Eq.(94)] probability for states with at least $N-M+1$ channels empty of quanta, states excluded by definition.

Again to get a simple expression suppose that all $N$ channels are similar so that all the $Y$ 's are equal. Then the equation collapses to

$$
Y^{N}-\left(\begin{array}{c}
N \\
M-1
\end{array}\right) Y^{M-1}=1
$$

Since we are assuming large $N$ and $M$, the right hand side of (117) is plainly negligible $(Y>1)$. Approximating the factorials in the combinatoric symbol with help of Stirling's formula, we can cast this equation in the form

$$
\ln Y \approx G(r) \equiv r(1-r)^{-1}|\ln r|+|\ln (1-r)|,
$$

which replaces Eq.(113).

Since $Y \equiv \prod_{j}\left(1-e^{-\mu \epsilon_{j}}\right)^{-1}$, we see that to obtain $\mu$ we merely have to replace the $\ln 2$ term in (114) by $G(r)$. Thus $\mu$ becomes a definite single valued function of $r$. It has to be calculated numerically by performing the sum in (114). Writing the final result as

$$
\dot{I}_{\max } \leq \alpha(r) \frac{E}{2 \pi \hbar}
$$

we find that for $r=\left\{10^{-3}, 10^{-2}, 10^{-1}, 1 / 2\right\}, \alpha=\{4.85,2.95,1.41,0.645\}$. The notable feature here is that $\dot{I}_{\max }$, and likewise $\epsilon_{\min }$, do not depend directly on the number of channels $N$, but only on the fraction of them which are required to contain quanta in order to certify arrival of a signal. This, of course, justifies our way of implementing the spatially blurred communication system. It can also be seen that for a blurred communication channel, the usual linear bound is correct except for a weak dependence of the coefficient on the filling fraction. In this sense Bremermann's claim ${ }^{11}$ that multichannel communication is subject to the linear bound is on the mark.

\section{Limitations on Information Storage}

The past two decades have witnessed a breakthrough in computer and data storage technology; one advance has been the great reduction in size of information storage devices. According to this trend individual atoms or molecules may one day become short term information-storage devices. Can this trend continue indefinitly, or is there is some physical limitation on the size devices of given information capacity may 
reach in the future? It seems plausible that as the size of information storage devices approaches elementary particle proportions, the end must come to the miniaturization process.

Now the maximum entropy for a system quantifies the maximum information that may be coded using all its microscopic degrees of freedom. Therefore, regardless of details of precisely how and where the information is held, a bound on entropy like (85) limits the maximum amount of information that may be inscribed in and retrieved from a physical system in terms of its maximum linear size and its energy. This bound is in harmony with the intuitive feeling that the entropy of a physical system must be limited by the available volume in phase space which, in turn, ought to depend on the system's dimensions and proper energy. But how sure are we of the correctness of the bound?

\subsection{Drawbacks of the Canonical Ensemble Method}

Originally inferred from considerations of black-hole physics, ${ }^{19}$ the bound was immediately subjected to scrutiny from the point of view of statistical physics. Early microcanonical numerical calculations of the specific entropy of free quantum fields confined to cavities of various shapes were carried out by Gibbons ${ }^{42}$. These, and later more extensive ones, ${ }^{13}$ have supported the bound in every case. In order to obtain more generic results, one of us applied in detail the canonical approach of statistical physics to quantum fields confined in various cavities. ${ }^{19,44}$ In the canonical approach (system parametrized by temperature or mean energy) the validity of the bound hinges on the sign and the value of the vacuum (Casimir) energy. If this last is positive and not very small on the scale of the typical mode frequencies, then the bound is obeyed with the maximum $H / E$ occurring at low excitation energy. ${ }^{19,44}$ However, field theoretic calculations for various cavities and fields frequently show that the vacuum energies are negative ${ }^{45-47}$ so that violations of the bound can occur at sufficiently low temperatures. Even if the vacuum energy vanishes exactly, or if one chooses to interpret the $E$ as the excitation energy above the vacuum, it is easy to see that violation of the bound is possible at low temperatures. According to the canonical ensemble the ratio $H / E \approx T^{-1}$ at low $T$, so that the bound is violated at sufficiently small temperature. ${ }^{47-49}$ although the energy range over which the violation occurs is extremely narrow. ${ }^{44}$

The very significance of canonical results in this regime is put into question by the observation that at low temperatures fluctuations are so large that mean energy is not a good indicator of actual energy. Recall that the ratio of a system's energy fluctuation $\Delta E$ to its mean energy $\bar{E}$ is $\Delta E / \bar{E} \approx N^{-1 / 2}$, where $\bar{N}$ is the mean number of quanta. At low $\bar{N}$ the energy fluctuations could be larger than the mean energy itself. Put another way, at low excitations the customary equivalence between canonical and microcanonical ensembles cannot be relied upon. Now the canonical ensemble owes its popularity more to the convenience it affords in calculations (which are always much more complicated, if not hopeless, in microcanonical ensemble), than to the conviction that it gives a more correct entropy. Whereas the microcanonical ensemble method relies only on very general assumptions like ergodicity, the canonical ensemble may be deduced from it only on basis of additional hypothesis like the validity of saddle point approximation, positivity of specific heat, etc. Sometimes the canonical ensemble fails entirely: the hydrogen atom cannot be canonically described. Therefore, the microcanonical approach appears to be the primary theoretical framework. Henceforth we conduct our discussion using microcanonical methods.

\subsection{One Particle Information Storage - Examples}

In the simplest instance information can be stored in a one-particle system by virtue of the multiplicity of quantum states. In an early investigation of bound (85) in microcanonical ensemble, Qadir ${ }^{20}$ considered a single free quantum mechanical particle confined to a volume $V$ and having energy up to $E$. Using the uncertainty principle to determine how many states are available, he took the logarithm of this quantity as the entropy of the system. He concluded that $H \sim \ln (E R)$ where $R \approx V^{1 / 3}$. This is consistent with bound (85). Perhaps a more interesting problem concerns a particle subject to some attractive force. Then the energy on the right hand side of the bound is decreased while the entropy is not necessarily affected, so that one tests the bound under more severe conditions. Additionally, this problem brings us closer to more realistic information storage systems.

If a molecule could be harnessed as an information storage device, the coding would have to exploit the multiplcity of available molecular levels. Because these states usually differ in energy, it is relevant to ask what is the maximum information which may be encoded for a given available energy. Suppose we apply the bound (85) taking care to include in $E$ all the energies, i.e., rest energies as well as excitation energies. Of 
course, in real atoms and molecules most of the energy is rest energy, and so (85) predicts, for typical atomic (molecular) dimensions and masses, that the limit is some $10^{6}$ bits. This certainly exceeds the logarithm of the number of atomic (molecular) states below ionization in known atoms and molecules, so bound (85) is easily satisfied. The seemingly discrepant case of the hydrogen atom with its infinity of levels is easily accounted for by remembering that the highly excited (Rydberg) states correspond to dimensions large by atomic standards. But it is interesting to consider an hypothetical atomic systems whose constituents' rest masses coould be adjusted at will. Would not reduction of such masses eventually bring (85) into conflict with the actual value of $(H / E)_{\max }$ ?

To elucidate this question we now consider, following Ref.19, the cumulative number of states $N(E)$ up to energy $E$ for one-particle quantum-mechanical systems described by Schrödinger's equation. Our examples are meant to capture the essential features of the electronic, rotational, and vibrational degrees of freedom we meet in atoms and molecules. We want to see whether the peak value of $(H / E)_{\max }$ is indeed bounded by $2 \pi R / \hbar c$, as predicted by (85). As expected, the inclusion in $E$ of the rest energy of the particle, however small, is essential for the bound to be obeyed, so we choose the zero of the energy scale accordingly.

\subsubsection{Particle in a One Dimensional Potential Well}

Our first example concerns a particle of mass $m$ in a one-dimensional potential well. Superconducting quantum interference devices (SQUIDs) can be modelled as particle-in-well systems. Let us assume that the particle is constrained to a range of radius $R$ on either side of an appropriately chosen point, regardless of its energy $E$. A simple way to count the number of states $N$ up to and including $E$ is to use the WKB formula ${ }^{36}$

$$
\int_{x_{\min }}^{x_{\max }} \sqrt{2 m(E-V(x)} d x=2 \pi \hbar(N+1 / 2),
$$

where $V(x)$ is the potential and $x_{\min }, x_{\max }$ are the roots of $V(x)=E$. Evidently, only the whole part of $\mathrm{N}$ given by (106) is meaningful. For the moment we ignore the inherent inaccuracy of the WKB formula for low-lying states.

Evidently, the range of $x$ is less than $2 R$. Further, $E-V \leq e$ where $e$ is the energy measured with respect of the bottom of the potential well. Thus,

$$
N \leq \frac{\sqrt{2 m e} R}{\pi \hbar}
$$

It is also clear that $E=e+m c^{2}$. Defining the dimensionless quantities $e_{*}=e / m c^{2}$ and $R_{*}=R m c / \hbar$ we have

$$
\frac{\ln N(E)}{E} \leq \frac{Y R}{2 \hbar c} ; \quad Y \equiv \frac{\ln \left(2 e_{*} R_{*}^{2} / \pi^{2}\right)}{R_{*}\left(1+e_{*}\right)} .
$$

It is clear that within the Schrödinger theory we can only consider the case $e_{*}<1$ (nonrelativistic particle). Let us now maximize $Y$ with respect to $e_{*}$. The maximum occurs at the $e_{*}$ determined by

$$
2 R_{*}^{2} e_{*}^{2}=\pi^{2} e_{*} \exp \left(1+1 / e_{*}\right),
$$

and amounts to $\left(R e_{*}\right)^{-1}$. Because $e_{*}<1$, the right hand side of (123) is never smaller than 72.93 and so $Y<0.1656$. Therefore, after transforming to bits,

$$
I<0.119 \frac{E R}{\hbar c} \text { bits }
$$

for all $e$. Thus a particle confined to a potential well satisfies bound (85) regardless of the choice of $m$.

\subsubsection{Rigid rotator}

Consider now a two-dimensional system, a rigid rotator with moment of inertia $I$ and mass $m$ confined within a sphere of radius $R$. This can serve to model the rotational levels of a molecule ( $m$ is the molecular mass), a futuristic information recorder. The rotational energy levels are given by $e=j(j+1) \hbar^{2} / 2 I$ with $j=0,1, \ldots$ labeling angular momentum; the levels are $2 j+1$ degenerate. The total energy is $m c^{2}+e$. Obiously, $N(E)$ is just the sum of $2 j+1$ from $j=0$ to the largest $j$ for which $m c^{2}+e$ does not exceed $E$. Denoting this by $j_{*}$ we find $N(E)=\left(j_{*}+1\right)^{2}$. Now, we are interested in the peak value of $\ln N(E) / E$. This obviously occurs for an $E$ which is a rotational level (if $E$ is increased slightly, the factor $E$ depresses the 
ratio while $N(E)$ does not grow unless the next level has been reached.) Thus with the notation $I_{*}=I / m R^{2}$ and $R_{*}=R m c / \hbar$ we may put

$$
\frac{\ln N(E)}{E} \leq \frac{2 X R}{\hbar c} ; \quad X=\frac{\ln \left(j_{*}+1\right)}{R_{*}+j_{*}\left(j_{*}+1\right) / 2 I_{*} R_{*}} .
$$

As a function of $j_{*}, X$ peaks at the $j_{*}$ determined by

$$
\left(2 j_{*}+1\right)\left(j_{*}+1\right) \ln \left(j_{*}+1\right)-j_{*}\left(j_{*}+1\right)=2 I_{*} R_{*}^{2}
$$

and

$$
X_{\max }=2 I_{*} R_{*}\left(j_{*}+1\right)^{-1}\left(2 j_{*}+1\right)^{-1} .
$$

Of course, if (112) does not give integral $j_{*}$, then $X_{\max }$ cannot be quite reached, and (127) actually gives us an upper bound on $I_{*} R_{*}$ for specific $j_{*}$. However, if $I_{*}$ and $R_{*}$ are so adjusted that the peak can be reached and $j_{*}=0,1,2,3, \ldots$, then $I_{*} R_{*}=0,1.08,5.24,13.4, \ldots$ with the increasing trend continuing indefinitely. Because the radius of the gyration cannot exceed $R, I_{*}<1$ so we get upper bounds on $I_{*} R_{*}$ itself to substitute in (113). In this way we find, after converting the result to bits, that

$$
I<0.499 \frac{E R}{\hbar c} \text { bits. }
$$

This is in harmony with bound (85).

\subsubsection{Three-dimensional harmonic oscillator}

Consider next a three-dimensional isotropic harmonic oscillator of rest mass $m$ and frequency $\omega$. This could model a defect in a crystal lattice used as an information cache. Its energy levels are

$$
e=\left(n_{1}+n_{2}+n_{3}+3 / 2\right) \hbar \omega
$$

where $n_{i}=0,1, \ldots \quad$ Again, the total energy is $E=m c^{2}+e . N(E)$ is evidently the number of ways in which the $n_{i}$ can be added in such a way that the total energy does not exceed $E$. Again, the peak $\ln N(E) / E$ is reached when $E$ exactly corresponds to some energy level. Let $F(n)$ be the number-theoretic function giving the number of ways in which three labeled non-negative integeres may be added to give the integer $n$. Then

$$
\frac{\ln N(E)}{E} \leq K \frac{R}{\hbar c}
$$

with

$$
K \equiv \frac{\ln F(n)}{R_{*}[1+(n+3 / 2) y]},
$$

where $y \equiv \hbar / m c^{2}$ and $R_{*}$ is defined as before.

The effective radius of the oscillator can be taken as the oscillation amplitude given by the virial theorem, $R^{2}=e / m \omega^{2}$, or equivalently

$$
R_{*}=(n+3 / 2)^{1 / 2} y^{-1 / 2} .
$$

Since $F(n)<(n+1)^{3}$, our problem reduces to finding the maximum of

$$
K=\frac{3 y^{1 / 2} \ln (n+1)}{(n+3 / 2)^{1 / 2}[1+(n+3 / 2) y]}
$$

with respect to $y$ and $n$. As a function of $y, K$ peaks at $y=(n+3 / 2)^{-1}$, i.e., where the oscillator's energy just equals the rest energy. Although this point is already outside the nonrelativistic domain, it should be clear that the formal peak value so obtained bounds the $K$ realizable by the nonrelativistic oscillator. And with $y$ optimized, the result peaks for $n=2$. Transforming the result to bits we have

$$
I<0.369 \frac{E R}{\hbar c} \text { bits. }
$$

Again this is in harmony with the bound (85) for all $m$ and $\omega$. 
Actually the range of applicability of our example transcends the harmonicity assumption. Any spherically symmetric potential well resembles a harmonic potential near the bottom. Since the peak of $\ln N(E) / E$ is reached at low excitation, it is likely that some anharmonicity of the potential does not change (134) much.

What is the moral of our examples? It is that, when the rest energy is included in the energy $E$, the number of states accessible to a quantum-mechanical system of size $R$ with energy limited to $E$ is less than $\exp (2 \pi E R / \hbar c)$. The inclusion of the rest mass in $E$ is essential. Without it any bound like (85) can be surpassed by adjusting parameters of the system, i.e., by making the moment of inertia of a molecule large. However, the rest energy can be made small by "molecular engineering" without upsetting our result. Since our examples can be tailored to electronic, vibrational, and rotational levels, we have just shown that the information that could be coded in an atom or molecule is indeed bounded by (85). For real atoms and molecules the maximum must fall considerably below (85). In fact, if we consider only electronic levels for which the electronic mass is the relevant one, then short of ionization bound (124) limits the information to a few tens of bits.

\subsection{Many Quanta Systems: Numerical Experiments}

Turn now to many particle systems. Kahn and Qadir ${ }^{21}$ investigated the number of states in noninteracting quantum mechanical many-particle systems. They counted available states by the semiclassical (continuum) approximation, and found support for bound (85), but expressed the opinion that the bound can only be strictly correct in that approximation. Now as is well known, the semiclassical approximation is poor for low lying states. It turns out (see below) that this is precisely the regime in which the ratio $H / E$ peaks. This makes it clear that the semiclassical approximation is not particularly well suited to analyze the bound. As will become clear in this section and in Sec.5.4., the bound is an exact result in the full quantum treatment.

Following Ref.13, we consider here not quantum mechanical many-particle systems, but rather relativistic quantum free field systems. There are several reasons. First, the extension to relativistic fields does not incurr extra computational challenges in the absence of interactions. Second, quantum fields provide a realization of black-body radiation which, being a high entropy system, is a prime challenger of bound (85). ${ }^{19}$ The third reason concerns the computational process. In considering ways to optimize computers, a useful reference would be a computing machine, itself composed of elementary quanta, in which information is coded in the occupation numbers of the various modes, and in which the elementary operations consist of shifting quanta from one mode to another. It is difficult to believe that any forseeable computer composed of macroscopic components could be more energetically efficient, or faster at storing, retrieving, or processing information. Thus, it is interesting to assess the information capacity of an assembly of quanta or, equivalently, the maximal entropy for given available energy.

We thus consider a collection of quanta of some field confined inside a cavity of some shape. The stationary one-particle modes of the system will have a discrete spectrum $\left\{\epsilon_{j}\right\}$ and to each mode $j$ there will correspond a degeneracy $g_{j}$. In view of indistinguishability of quanta, a many-quanta state is specified fully by the occupation numbers $\left\{n_{j}\right\}$ of the various modes. If there are no interactions, the energy of the state is $\sum n_{j} \epsilon_{j}$.

Let $\Omega(E)$ represent the number of distinct quantum states accessible to the system with energy (measured from the vacuum) not exceeding $E$. We assume the vacuum in nondegenerate so that $\Omega(0)=1$. If the quanta are (indistinguishable) bosons, the number of ways to realize a set of occupation numbers $\left\{n_{j}\right\}$ is ${ }^{34}$

$$
D\left\{n_{j}\right\}=\prod_{i} \frac{\left(n_{i}+g_{i}-1\right) !}{n_{i} !\left(n_{i}-g_{i}\right) !} .
$$

For fermions the exclusion principle eliminates a number of possibilities so that $D\left\{n_{j}\right\}$ is smaller. As we raise the energy a jump of $\Omega(E)$ equal to $D\left\{n_{j}\right\}$ occurs as $E$ coincides with some $\sum n_{j} \epsilon_{j}$. Then $\Omega(E)$ stays constant until the next such coincidence. Therefore, $\Omega(E)$ looks like a ladder function. Ordinarily entropy is defined as the logarithm of the function $D\left\{n_{j}\right\}$. Since this is a very discontinous "comb" function, we prefer to take $S=\ln \Omega(E)$, as done by Gibbons in his early numerical experiments. ${ }^{42}$ This agrees with Eq.(2).

Evidently for a many-modes field, $\Omega(E)$ is a very complicated combinatoric function. It is hopeless to try to calculate it analytically. Here we describe numerical experiments carried out in Ref.13 which extended Gibbons' early ones. The procedure adopted for bosons was the following:

- List the energies $\epsilon_{j}$ of the modes and their degeneracies $g_{j}$. Only massless fields were considered; massive quanta "waste" energy in the rest mass which could have been used to reach more states, and their $\Omega(E)$ can only be smaller. 
- Populate the modes according to a pattern which guarantees inclusion of all many-quanta states up to some energy ceiling. We describe here the pattern used for Bose quanta; fermions are further constrained by the exclusion principle and thus their $\Omega(E)$ must be lower.

- Bin the many-quanta states found in narrow energy bins, and deduce an approximation to $\Omega(E)$.

The populating strategy was the following. First a single quantum was succesively promoted through the modes with $\epsilon_{j}$ increasing until it exceeded the ceiling. The number of states appearing at each stage of the promotion was computed from Eq.(135) and these numbers were binned by energy. Then the first quantum was returned to the lowest lying mode, and a second quantum was added to that mode. Next the first quantum was promoted mode by mode until the energy ceiling was reached. At this point the second quantum wss promoted by one mode, and the first was returned to that same mode. Then the first quantum was promoted again in the previous pattern. Numbers of states were computed from (135) at each promotion and binned by energy. When promotion of the second quantum with return of the first to that same mode already led to energy in excess of the ceiling, a third quantum was added at the lowest lying mode and the first two quanta were returned to it. The pattern of promotions, first quantum first, was repeated. At each stage a new quantum would be added. When addition of a new quantum and return of the previous quanta to the lowest lying mode caused the energy to exceed the ceiling, the process was stopped. This populating pattern assures that all quantum configurations allowed by the principle of indistinguishability are counted. A count of the number of states accumulated in all bins beneath energy $E$ gave an approximation for $\Omega(E)$. This function was thus reconstructed up to the chosen ceiling.

Fig.3. Specific entropy vs. energy for a scalar field in a rectangular box of dimensions 1x0.95x0.9 with Neumann boundary conditions. Numerical values assume $\hbar=c=1$.

The spectra for fields in various cavities were described in Ref.19, and are summarized in Ref.13. In a spherical cavity of radius $R$ the generic form of the spectrum is

$$
\epsilon_{j}=\frac{\hbar c j_{n \ell}}{R} .
$$

For the scalar field with Dirichlet boundary conditions $j_{n \ell}$ means the $n$-th zero of the spherical Bessel function of order $\ell ; \ell=0,1,2, \ldots$ and the modes are $(2 \ell+1)$-fold degenerate. For Neumann boundary condition $j_{n \ell}$ is to be interpreted as the $n$-th zero of the derivative of the spherical Bessel function of order $\ell$ (degeneracies and range of $\ell$ are as for the Dirichlet case). The neutrino field in a sphere can also be analyzed under a special boundary condition ${ }^{19}$ giving the spectrum (136) based on the zeroes of the spherical Bessel functions, each with degeneracies $2(2 \ell+1)$; again $\ell=0,1,2, \ldots$ For the electromagnetic field in a highly conducting cavity, the tangential electric field must vanish on the boundary. The spectrum is then of the form (136) 
with $\ell=1,2,3 \ldots \quad$ except that for each value of $\ell$ there are eigenvalues corresponding to zeroes of both the spherical Bessel function and of its derivative. Each of these is $2 \ell+1$ degenerate.

In a rectangular cavity with sides $A, B$ and $C$ the mode energies of both fields are

$$
e=\pi \hbar\left(\frac{i^{2}}{A^{2}}+\frac{j^{2}}{B^{2}}+\frac{k^{2}}{C^{2}}\right)^{1 / 2}
$$

For the scalar field with Dirichlet boundary conditions $i, j, k=1,2,3, \ldots$ and the modes are nondegenerate (excepting accidental degeneracy arising from conmensurate $A, B$ or $C$ ). For the electromagnetic field with vanishing tangent electric field on the boundary, $g_{i j k}=2$ for $i, j, k=1,2,3, \ldots$ and $g_{i j k}=1$ when one of $i, j$ or $k$ vanishes. Modes with two or three vanishing quantum numbers are excluded. (Higher degeneracies are possible only when some of the sides are conmensurate).

In the numerical experiments the energy ceiling was set at some seven times the value of the lowest mode energy. This range included some 50-200 modes (not counting degeneracies) in most cases. The number of quantum states so included was of order $10^{4}$. Some 200 energy bins were used which provided sufficient resolution. An example of the detailed behavior of $\ln \Omega(E)$ is seen in Fig.3.

Generically $\ln \Omega(E)$ starts at zero for $E=0$, and rises with $E$ in an oscillatory fashion but faster, on average, than linearly. Then the rise rate moderates and $\ln \Omega(E)$ tends asymptotically to a $E^{3 / 4}$ behavior. This last is easily understood. For large energy there are many possible states so that the thermodynamic limit sets in: the collection of quanta behaves like black body radiation. Since for black body radiation the energy $E \propto T^{4}$, but the entropy $H \propto T^{3}$, we have $\ln \Omega(E)=H \propto E^{3 / 4}$. At any rate, it is clear that the specific entropy $\ln \Omega(E) / E$ must always have an absolute peak at some not too large $E$. This is certainly consistent with bound (85). Values of the peak $H / E$ were obtained numerically in a number of examples by scanning the numbers stored in the bins, and some are displayed in Table 1 . It may be seen that they always comply with bound (85).

Table 1. Peak specific entropy: numerical result, analytic estimate and bound (85).*

\begin{tabular}{llllll}
\hline Field & Cavity & Boundary & $(H / E)_{\max }$ & $\zeta(4)^{1 / 4}$ & $2 \pi R$ \\
\hline scalar & unit sphere & Dirichlet & 0.454 & 0.476 & 6.283 \\
$"$ & $"$ & Neumann & 0.701 & 0.721 & 6.283 \\
electromagnetic & $"$ & conducting & 0.716 & 0.749 & 6.283 \\
neutrino & $"$ & see Ref.19 & 0.547 & 0.566 & 6.283 \\
scalar & $1 \times 1 \times 1$ & Dirichlet & 0.269 & 0.280 & 5.441 \\
$"$ & $1 \times 0.95 \times 0.9$ & Dirichlet & 0.249 & 0.266 & 5.174 \\
& $"$ & Neumann & 0.428 & 0.457 & 5.174 \\
electromagnetic & $"$ & conducting & 0.365 & 0.380 & 5.174 \\
scalar & $1 \times 0.66 \times 0.2$ & Dirichlet & 0.122 & 0.131 & 3.828 \\
& & & & & \\
\hline
\end{tabular}

${ }^{*}$ Largest dimension of rectangular cavities taken as unit of lenght; here we take $\hbar=c=1$.

\subsection{Many Quanta Systems: Analytic Results}

Numerical examples dealing with specific cavity geometries cannot prove the bound. Therefore, we discuss two analytic results that go a long way to establishing its validity.

\subsubsection{Analytical Estimate of Peak Specific Entropy}

An approximate proof of bound (85) in microcanonical ensemble can be based on an analytic argument developed in Ref.13. This shows that the peak value of $H / E$ can be approximated by $[\zeta(4)]^{1 / 4}$, where $\zeta(\kappa)$ is an analogue of the Riemann zeta function constructed from the mode spectrum of the field:

$$
\zeta(\kappa)=\sum_{j} \epsilon_{j}^{-\kappa}
$$

In three dimensional space the number of eigenvalues below $E$ grows as $E^{3}$; therefore the sum representing the zeta function converges only for $\kappa>3$. If all we desire is an upper bound on $H(E)$ of a given system, the 
rule $(H / E)_{\max } \approx[\zeta(4)]^{1 / 4}$ is a great work saver. It usually overestimates $(H / E)_{\max }$ by only a few percent [see Table 1 and also Ref.13]. Therefore, in most cases the information codable in a quantum system whose interactions can be neglected is analytically approximated by

$$
(H / E)_{\max } \approx[\zeta(4)]^{1 / 4} .
$$

The analytic estimate (139) and bound (85) are displayed for a few examples in Table 1.

When only a rough estimate of $\zeta(4)$ is required, it is usually sufficient to cut off the sum in (138) after a few terms. This is because the terms in $\zeta(4)$ drop off rapidly. Also the function is raised to power $1 / 4$ so errors in it are diluted. In this approximation it is easy to see why a bound of form (85) must hold. On dimensional grounds the first eigenvalue $\epsilon_{1}$ has to be of order $\hbar c R^{-1}$ for a massless field, where $R$ is the radius of the circumscribing sphere. Thus $[\zeta(4)]^{1 / 4}$ must be a few times $R / \hbar c$. Then to within a numerical factor (139) reduces to (85).

\subsubsection{Microcanonical Proof of Bound on Specific Entropy}

We now present an exact proof of bound (85), a much simplified and more rigorous version of that given in Ref.13. Our system consists of a free field (scalar, electromagnetic, ... ) confined within a cavity of arbitrary shape by appropriate boundary condition. The mathematical framework we shall use is that developed in Sec.4.3 for pulsed communication. Recall that instead of trying to calculate the cummulative number of quantum states $\Omega(E)$ up to a given energy budget $E$, we switched attention to the auxiliary quantity $N(E)$ which overestimates $\Omega(E)$. $N(E)$ was shown to be the solution of the integral equation (106) in which the number of modes with $\epsilon_{j}=\hbar \omega_{j} \leq E, n(E)$, enters in the kernel. In our one dimensional problem in Sec.4.3 we knew the spectrum $\left\{\hbar \omega_{j}\right\}$; here each cavity will have a different one-quantum spectrum.

For concreteness think of a scalar field $\psi$. We are then interested in solving the eigenvalue problem

$$
-\nabla^{2} \psi_{j}=\omega_{j}^{2} \psi_{j}
$$

subject to the general boundary condition

$$
\left[\frac{\partial \psi}{\partial n}+\alpha \psi\right]_{\partial \Sigma}=0
$$

where $\Sigma$ is the domain of the cavity and $\partial \Sigma$ its boundary; $\partial / \partial n$ represents the normal derivative at $\partial \Sigma$. For $\alpha=0$ (141) corresponds to Neumann boundary conditions; for $\alpha \rightarrow \infty$ it reduces to Dirichlet boundary conditions. For generic $\alpha$ we have Robin boundary conditions.

Since it is hopeless to try to deal with the problem on a cavity-by-cavity basis, we ask what generic properties of the spectrum can be inferred by solving for the spectrum corresponding to a fictitious spherical cavity that completely encloses the true system. This last is a much simpler problem, and there turns out to be a simple relation between the special result for it and the generic result.

We first rephrase the eigenvalue problem as a problem in the calculus of variations which provides a powerful method for comparing eigenvalues for related problems. Define the functional $^{50}$

$$
\omega^{2}[\chi]=\frac{\int_{\Sigma} \nabla \chi \cdot \nabla \chi+\alpha \int_{\partial \Sigma} \chi^{2}}{\int_{\Sigma} \chi^{2}}
$$

The extremal values obtained when $\omega^{2}[\chi]$ is varied with respect to $\chi$ are known to reproduce the eigenvalue spectrum of problem (140). If $\alpha=0$ and the variation is performed with $\chi=0$ on $\partial \Sigma$, one gets the Dirichlet spectrum. If instead free variation of $\chi$ on $\partial \Sigma$ is allowed, one gets the Neumann spectrum. ${ }^{50}$ Taking $\alpha \neq 0$ and allowing free variation of $\chi$ at $\partial \Sigma$ gives the Robin spectrum. The minimum of $\omega^{2}[\chi]$ is the lowest eigenvalue $\omega_{1}$ of the corresponding boundary value problem, and to it corresponds the ground state eigenfunction $\chi_{1}$. This eigenfunction may thus be approximated by substituting a very flexible trial function in the functional. Higher eigenvalues $\omega_{j}$ and eigenfunctions $\chi_{j}$ are obtained by varying the above functional with a trial function $G$ orthogonal to all those obtained previously, i.e., $\int_{\Sigma} G \chi_{k}=0$ for all $k<j$.

There is an alternative way to characterize the sequence of eigenvalues variationally: the minimax principle. ${ }^{50}$ The $i$-th trial function for minimizing $\omega^{2}[\chi]$ is chosen as satisfying the appropriate boundary conditions and orthogonal to any set of $i-1$ independent functions $G$ with the same boundary conditions. 
Then the minimum obtained is maximized over all choices of the $G$ 's and there result $\omega_{i}^{2}$ (the $i$-th eigenvalue in magnitude) and the corresponding eigenfunction $\chi_{i}$. According to the minimax principle if $\bar{\omega}_{i}$ is the $i$-th eigenvalue of a variational problem in which the trial functions belong to a certain class of admissible functions $\{\bar{G}\}$ e.g. all continuous and differentiable functions satisfying one of conditions (141) on the cavity boundary, and $\omega_{i}$ is the $i$-th eigenvalue for the same problem with respect to a second class of functions $\{G\}$ which are subject to additional constraints, then $\bar{\omega}_{i} \leq \omega_{i}$. In other words, adding constraints to the trial functions can only lift all the spectrum.

In our case the class of trial functions for the real cavity will be required to vanish not only at the spherical cavity surface, but also in the region between the real and the reference spherical cavities. Because of the additional constraint,

$$
\bar{\omega}_{i} \leq \omega_{i}
$$

where $\bar{\omega}_{i}$ now denotes the eigenfrequency for the sphere corresponding to $\omega_{i}$ of the real cavity.

Now recall the zeta function defined by (138). We evidently have the inequality ${ }^{42}$

$$
\zeta(\kappa) \omega_{j}^{\kappa}=\sum_{i=1}^{\infty}\left(\frac{\omega_{j}}{\omega_{i}}\right)^{\kappa}>\sum_{i=1}^{j}\left(\frac{\omega_{j}}{\omega_{i}}\right)^{\kappa}>j
$$

since in the right hand side there are precisely $j$ terms no smaller than unity. Let us now define a "reference" spectrum,

$$
\omega_{j}^{*} \equiv\left[\frac{j}{\bar{\zeta}(\kappa)}\right]^{1 / \kappa}
$$

where $\bar{\zeta}(\kappa)$ is the zeta function for the spherical cavity. According to $(143) \bar{\zeta}(\kappa)>\zeta(\kappa)$ so that by (144) the reference "spectrum" must satisfy

$$
\omega^{*}{ }_{j} \leq \omega_{j} .
$$

Now since the reference spectrum is everywhere lower than the true spectrum, the corresponding cummulative number of quantum states $\Omega^{*}(E)$ for the former must satisfy

$$
\Omega(E)<\Omega^{*}(E)
$$

(a given energy $E$ can be split in more ways if all the mode energies are lower).

In entire analogy with $N_{m}(E)$ of Eq. $(102)$, it is now possible to define $N^{*}{ }_{m}(E)$ by simply replacing the true mode spectrum by the reference one. If we now sum over number of quanta as in Eq.(104), we get $N^{*}(E)$ which must evidently bound $\Omega^{*}(E)$ from above. In view of (147) we thus have

$$
H_{\max }(E)=\ln \Omega(E)<\ln N^{*}(E) .
$$

The $N^{*}(E)$ is the solution of the integral equation (106) with the reference mode number density $d n^{*}(E) / d E$ as kernel. Definition (145) is equivalent to

$$
n^{*}(E) \equiv \bar{\zeta}(\kappa) E^{\kappa}
$$

We proceed to solve the integral equation $(106)$ for $N^{*}(E)$ by Laplace transforms. Since the Laplace transform of $n^{*}(E)$ is

$$
\widetilde{n}^{*}(s)=\Gamma(\kappa+1) \bar{\zeta}(\kappa) s^{-(\kappa+1)},
$$

we have in analogy with Eq.(107)

$$
\widetilde{N}^{*}(s)=\frac{s^{\kappa-1}}{s^{\kappa}-\kappa ! \bar{\zeta}(\kappa)} .
$$

$N^{*}(E)$ is to be obtained by inverting the Laplace transform $\widetilde{N}^{*}(s)$, namely, by evaluating the integral of $\widetilde{N}^{*}(s) \exp (s E) / 2 \pi i$ along a contour parallel to the imaginary axis to the right of the $\kappa$ poles of $\widetilde{N}^{*}(s)$. These poles are distributed uniformly around a circle in the complex $s$-plane with radius $(\kappa ! \bar{\zeta}(\kappa))^{1 / \kappa}$. Their phases are those of the $\kappa$ distinct $\kappa$-th roots of unity $\sigma_{1}, \sigma_{2}, \ldots, \sigma_{\kappa}$. It is convenient to translate the contour to large negative $s$ while indenting it to avoid the poles as illustrated in Fig.4 for the concrete case $\kappa=4$. 
In this way only the residues contribute to the inverse transform; the contribution of the vertical part of the contour vanishes in the limit of large negative real part of $s$. In view of this,

$$
N^{*}(E)=\kappa^{-1} \sum_{n=1}^{\kappa} \exp \left\{\sigma_{n}[\kappa ! \bar{\zeta}(k)]^{1 / \kappa} E\right\}
$$

Fig.4. Contour for evaluating the inverse Laplace transform in Eq.(151).

Since the zeta function is only defined for $\kappa>3$, let us choose $\kappa=4$ [this leads to the tightest bound on $\Omega(E)$ ] and set $x \equiv(4 ! \bar{\zeta}(4))^{1 / 4}$. Then by exploiting various trigonometric and transcendental identities ${ }^{51}$ we have

$$
\begin{gathered}
N^{*}(E)=\frac{\exp (x E)+\exp (-x E)+\exp (i x E)+\exp (-i x E)}{4} \\
=\frac{\cosh (x E)+\cos (x E)}{2} \leq \frac{\cosh (x E)+1}{2} \\
=\cosh ^{2}(x E / 2) \leq \exp (x E)=\exp \left\{[4 ! \bar{\zeta}(4)]^{1 / 4} E\right\} .
\end{gathered}
$$

According to (148) the specific entropy should satisfy,

$$
\frac{H(E)}{E}<[4 ! \bar{\zeta}(4)]^{1 / 4}
$$

We see that the rigorous bound here obtained for $H / E$ is just a factor of 2.2 above our analytic estimate, Eq.(139). According to the argument following Eq.(139), the bound is seen to be of the same form as (85), and amounts to a proof of it.

Neumann boundary conditions for the scalar field raise an interesting question. The lowest eigenvalue is $\omega_{1}=0$ with corresponding homogeneous eigenfunction. As a result the formal zeta function is always infinite, and bound (154) is uninteresting. We have argued elsewhere ${ }^{13,23,52}$ that "zero modes" like this one have to be excluded from consideration when calculating the entropy because they correspond to a field condensate analogous to the superfluid condensate, not to modes that can be populated by a definite number of quanta. It follows that for consistency, zero modes must be excluded from the zeta function, a procedure that was followed in constructing the fifth column of Table 1 . Unruh ${ }^{53}$ has argued that a zero mode furnishes the opportunity to build an infinity of quantum states with the same energy, and thus violates bound (85). In fact the states dispayed by Unruh can be understood, in analogy with the phenomenon of symmetry 
breaking, to belong to different systems. ${ }^{52}$ Thus they do not contribute to the entropy of one system. It must be realized that were the infinity of states truly possible, systems with a zero mode would be endowed with infinite entropy. There is simply no evidence from closely akin systems, e.g. superfluids, that this is the case.

Is bound (85) respected by systems other than the ones considered in Table 1. ? What about systems of fermions? We know that, because of the exclusion principle, a fermion with a single helicity will have smaller $(H / E)_{\max }$ than a boson with a single helicity having the same spectrum. Of course the Dirac equation has, in general, a different spectrum than, say, the scalar equation. The results quoted in Table 1 . for the neutrino field (the boundary condition is described in Ref.19) show that the fermion character does not, in this example, endanger the bound.. What about massive quanta? Because they put a goodly fraction of the energy in rest mass, rather than in "phase space", massive fields should give lower $(H / E)_{\max }$ than massless ones, provided the rest masses are included in $E$ as in Sec.5.2. So far the discussion has referred to one field. If we put in a cavity a sufficiently large number of distinct field species, the bound can be violated. ${ }^{48,54}$ This is because the zeta function for a mixture of $N$ fields with identical spectra is $N$ times the individual field's zeta function. Thus the estimate (139) rises as $N^{1 / 4}$, and must eventually surpass (85) (this usually happens only for hundreds of field species). However, the point has been made ${ }^{13,44}$ that since the number of fermion generations is limited (almost certainly only 3 ), the number of elementary particle species is below $10^{2}$. This is not enough to allow bound (85) to be surpassed. Indeed, the argument has been reversed to set bounds on the number of particle generations starting from the bound on specific entropy (85). ${ }^{55,56}$

\subsection{The Effects of Interactions}

Although the specific entropy bound is now well established for free fields, its status in the presence of interactions is not so clear. Interactions are important in many information storage and communication technologies, e.g. SQUIDs and nonlinear optical media. It is clear that interactions raise new challenges for bound (85). First, even weak interactions can lead to the formation of bound states, thus creating "new species". If enough of these form, the bound might be violated by the mechanism mentioned in Sec.5.4. Second, nonlinear interactions are bound to complicate the energy spectrum and this might lead to the overthrow of the bound. Finally, in the presence of interactions the additivity of energies of quanta which was crucial for the work in Secs. 5.2-5.4 falls through. Despite these hurdles quite a bit has been learned about the validity of the bound in the presence of interactions, and this is summarized in the following subsections.

\subsubsection{The Hadron System}

The blatant example in nature of manifold species arising from the binding of a few elementary components is the hadron spectrum with its myriad resonances. Therefore, an analysis was made of the number of quantum states in a hadron gas as a function of energy budget. ${ }^{13}$ It being hopeless to deal explicitly with the strong interactions, the bootstrap philosophy was adopted: the influence of the strong interaction is approximately accounted for by considering simultaneously the full complement of hadronic species. The hadronic spectrum is well described by Hagedorn's semiempirical density of levels which includes spin and isospin multiplicity as well as hadron-antihadron duplicity. If hadron energy $e$ is measured in MeV, Hagedorn's formula for the number of levels in the interval de is ${ }^{57,58}$

$$
\mu(e) \approx 26300\left(2.5 \times 10^{4}+e^{2}\right)^{-5 / 4} \exp (e / 160) d e .
$$

A realization of the hadron spectrum was constructed which agreed with (155). This gave the list of energy levels $\left\{\epsilon_{j}\right\}$ of the system. The levels were then populated according to the methods described in Sec.5.3. Kinetic energy was ignored (it is nonegligible unless the hadron gas is relativistic). The algorithms described in Sec.5.3. led to $(H / E)_{\max }=0.007 \mathrm{MeV}^{-1}$. The hadron gas must evidently be confined to a space with radius no smaller than $1 \times 10^{-13} \mathrm{~cm}$. Therefore, $2 \pi R / \hbar c>0.032 \mathrm{MeV}^{-1}$. We see that the hadron gas obeys the specific entropy bound (85). This example shows that even if many species can form because of interaction, they tend to be sufficiently separated in energy for the bound to be upheld.

\subsubsection{Solitons as Information Storage Devices}

Does nonlinearity by way of its effect on the spectrum allow violations of the bound? One of the simplest 
covariant models of self interacting field is the quartic self-interacting one; it obeys the equation

$$
\square \Phi-m^{2} \Phi+\lambda \Phi^{3}=0,
$$

where $\square$ is the d'Alembertian and $m^{2}$ and $\lambda$ are real positive constants, the last measuring the strength of the interaction. This equation has static self-confining field configurations. In one space dimension these are solitons. For example, in empty space Eq.(156) has, apart from the two "vacuum" stationary solutions $\Phi= \pm m \lambda^{-1 / 2}$, the time independent soliton solution

$$
\Phi_{0}(x)=\frac{m}{\sqrt{\lambda}} \tanh \frac{m x}{\sqrt{2}}
$$

which interpolates between the two vacuua. The soliton is a nonperturbative creature; it cannot be obtained by perturbation theory based on the vacuua. It has finite energy $\sqrt{8 / 9} m^{3} \lambda^{-1}$. ${ }^{59}$ Since only a fraction $3 \times 10^{-4}$ of the total energy lies in $x>\sqrt{8} \mathrm{~m}^{-1}$, we define the soliton radius as $R_{s}=\sqrt{8} \mathrm{~m}^{-1}$.

Note that the Lorentz invariance of Eq.(156) allows one to immediately obtain a traveling soliton solution by simple transformation. Thus the question of information storage is closely bound up with that of communication. Solitons are of more than academic interest here. When one burns a fingertip, the information is conveyed by a solitary wave of axon potentials traveling from finger to brain along the nervous fibers.

Our soliton offers a model for a self-contained information storage system. The possibility of storing information in the soliton arises because excitations of it are possible, thus providing a variety of states for information coding. Consider a small perturbation about the soliton configuration: $\Phi=\Phi_{0}(x)+\eta(x, t)$. It satisfies the linearized equation

$$
\square \eta-m^{2} \eta+3 \lambda \Phi_{0}^{2} \eta=0 .
$$

Look for eigenmodes of these equation of the form $\eta_{j}(x, t)=\Xi_{j}(x) e^{-i \omega_{j} t}$. The mode functions satisfy

$$
\omega_{j}^{2} \Xi_{j}=c^{2}\left[-\nabla^{2}+m^{2}-3 \lambda \Phi_{0}^{2}\right] \Xi_{j} .
$$

As usual they form a complete set and are orthogonal.

In the canonical quantization approach, the field operator corresponding to $\eta$ may be expanded as

$$
\hat{\eta}(x)=\sum_{j}\left[a_{j} \Xi_{j}(x)+a_{j}^{\dagger} \Xi_{j}^{*}(x)\right]
$$

where $a_{j}$ and $a_{j}^{\dagger}$ satisfy the canonical commutation relations for the harmonic oscillator. The field hamiltonian is

$$
H=\frac{1}{2} \int\left[\dot{\Phi}^{2}+(\nabla \Phi)^{2}-m^{2} \Phi^{2}+\frac{1}{2} \lambda \Phi^{4}\right] d^{3} x .
$$

Separating the terms quadratic in $\eta$, substituting (160), and normal ordering, one gets

$$
\hat{H}=\sum_{j} \hbar \omega_{j} a_{j}^{\dagger} a_{j}
$$

Accordingly, we interpret $a_{j}^{\dagger}$ and $a_{j}$ as creation and annihilation operators for quasiparticles "riding" on the soliton. To this approximation the energies of these quanta are additive.

With $\Phi_{0}$ of Eq.(157), (159) is Schrödinger's equation for a particle moving in the potential $V=-3 \operatorname{sech}^{2}(m x / \sqrt{ } 2)$, a standard problem in quantum mechanics. ${ }^{38}$ In our specific problem there are two bound states and a continuuum. ${ }^{59,60}$ The eigenvalues and eigenfunctions are summarized in Table 2 :

Table 2. Eigenvalues and eigenfunctions of soliton perturbations. ${ }^{*}$

\begin{tabular}{ll}
\hline eigenvalue & eigenfunction \\
\hline$\omega_{0}^{2}=0$ & $\Xi_{0}(z)=\operatorname{sech}^{2} z ;$ \\
$\omega_{1}^{2}=\frac{3 m^{2}}{2}$ & $\Xi_{1}(z)=\sinh z \operatorname{sech}^{2} z ;$ \\
$\omega_{k}^{2}=m^{2}\left(\frac{k^{2}}{2}+2\right)$ & $\Xi_{k}(z)=3 e^{i k z}\left(\tanh ^{2} z-\frac{1}{3}-\frac{k^{2}}{3}-i k \tanh z\right)$ \\
\hline${ }^{*} z \equiv m x / \sqrt{ } 2$ and $-\infty<k<\infty$ is a continuous index.
\end{tabular}


The zero mode is not useful for storing information; it does not correspond to a soliton excitation but is rather related to translational invariance ${ }^{59}$ as clear from the fact that $\Xi_{0}(x) \propto\left[\Phi_{0}(x+\delta x)-\Phi_{0}(x)\right] / \delta x$. Thus, it makes no sense to talk of quanta occupying this level. While the $\Xi_{1}$ mode is a bound state confined to the soliton's extent, the continuum levels $\Xi_{k}$ are not confined within the soliton radius $R_{s}$ but spread to infinity. Accordingly, if we wish to use the soliton to store information without help of a confining box, we can only use its first excitation $\Xi_{1}$.

This being clear, the number of possible information-holding configurations based on the soliton equals the number of quanta that might populate the first excited level. To this number we must add unity to account for the (background) soliton configuration itself. Unlike the situation for self-heralding signals where the ground state is not counted, here the background's existence can be detected, e.g. it carries energy. Therefore, one should include this contribution to the total number of states. Thus, the number of possible configurations within an energy budget $E$ above the (unexcited) soliton energy is $N(E)=1+\left[\left[E / \omega_{1}\right]\right]$ where, again, $[[x]]$ stands for the integral part of $x$. The information that may be stored is thus

$$
I_{\max }=\ln \left(1+\left[\left[\frac{E}{\omega_{1}}\right]\right]\right) \log _{2} e \text { bits. }
$$

Since $\ln (1+[[x]]) \leq x$ we find, in light of the definition of $R_{s}$ and the value of $\omega_{1}$, that

$$
I_{\max }<0.416 \frac{E R_{s}}{\hbar c}
$$

which is consistent with bound (85).

To exploit the continuum states for information storage, one must confine them (after all we are discussing storage in a finite space). One way to do this would be to put the soliton in a box. However, it would then be impossible to meet the boundary condition $\Phi_{0}=0$ at both ends. To get over this hurdle we might consider instead a soliton-antisoliton pair. (An antisoliton is the solution of the field equation differing from (157) only in sign.) We would put the soliton at one end of the box with its node $\Phi_{0}=0$ on the box wall, and we similarly locate the antisoliton at the other end with its node at the farther wall. Since the pair are well separated, they constitute a good approximation to a static exact solution of the equation. We would then go on to study excitations of the soliton-antisoliton system within the box. However, the stationary configuration envisaged is far from being a generic stationary configuration of the field theory in a box. What is the complete set of such configurations, and what do their excitations look like?

\subsubsection{Scalar Field with Quartic Self-Interaction in a Box}

Bekenstein and Guendelman ${ }^{22}$ investigated this issue by looking at the massless charged scalar field with self-interaction confined to a box. When the interaction is quartic the equation is (156) again, except that instead of $\Phi^{3}$ we must write $\Phi^{2} \Phi^{*}$. Classically this field exhibits a continuum of configurations within a finite interval of energy, so that it should violate bound (85). Quantum mechanically the situation is different: the requirement that configurations have integral charge discretizes the spectrum of stationary states. Each stationary state and the excitations of it form a particular charge sector. Within each sector permitted energies are sufficiently separated to give the bound a fighting chance.

The energy spectrum can be solved analytically in the case of a one-dimensional box. Call its size $L$. In one dimension the quartic coupling constant $\lambda$ is dimensional: $L_{*} \equiv(\hbar c \lambda)^{-1 / 2}$ is a scale of length. Bekenstein and Guendelman computed the lower spectrum of stationary state energy levels explicitly for the range of dimensionless box sizes $0.05<L / L_{*}<20$, and also obtained asymptotic formulae for box size outside this range. Although the spectrum is complicated, the levels are well spaced, and the quantity $\ln \Omega(E) / E$ behaves qualitatively as in Fig.3. The values of $(H / E)_{\max }$ are below the specific entropy bound, $2 \pi(L / 2) / \hbar c$, by a sizeable factor regardless of the value of $L / L_{*}$. Taking into account excitations within each charge sector does not increase the entropy much, and the bound (85) continues to be respected. This example shows that nonlinear interactions do not necessarily violate the bound on specific entropy even when they introduce extraneous scales into the problem and change the nature of the energy spectrum.

More complicated situations involving interacting fields in a box may be handled by path integral techniques. ${ }^{61}$ There is an indication that bound (85) will be respected for a large class of interactions.

\subsubsection{The Gravitational Interaction}

Does the gravitational interaction help to transcend the bound? After all, gravitation is highly nonlinear

and introduces a special scale of length, the gravitational radius. First we should mention that from the 
beginning ${ }^{19}$ it was clear that a nonrotating neutral black hole, the most bound of systems, just saturates bound (85). Wald, Sorkin and Jiu ${ }^{62}$ considered the entropy of a self gravitating sphere of black body radiation in equilibrium. They concluded that this would respect bound (85) provided the solution of the hydrostatic equilibrium equations is nonsingular. Singular solutions were considered by Zurek and Page ${ }^{63}$ who did not find any whose entropy exceeded that allowed by the bound. Admittedly, none of the results mentioned is very generic. However, it is interesting that systems in which highly nonlinear interactions play a major role do not show much predisposition for violating bound (85).

\subsection{Information Storage in One Dimension}

One dimensional information storage systems are quite important. In a magnetic tape information is basically stored in one dimension. The DNA molecule is a more striking example in which the sequencing of four types of molecules codes the genetic information. A more general system of this sort is one in which $N$ "molecules" picked from $n$ species are arranged in a chain. There are $n^{N}$ such sequences so that $H_{\max }=N \ln n$. If $m$ is the typical molecular mass, and $\varsigma$ is the typical molecular radius, the system's energy is $E=N m c^{2}$ and its half-length (if not curled up) is $L / 2=N \varsigma$. Of course, $\varsigma>\hbar / m c$ (a molecule must be larger than its Compton length). Therefore $2 \pi E(L / 2) / \hbar c>2 \pi N^{2}$. This far exceeds $H_{\max }$ unless the number of molecule species is exponentially large. Therefore, the system in question satisfies the information bound.

It is also possible to store information in the oscillations of the molecules about their equilibria (it is unclear whether this option is exploited in biological systems). Here interactions are quite important. To make headway in the analysis, we assume that it is possible to define normal modes for the oscillations, so that the excitations are free phonons. Phonons are characterized by momentum $p$ which may be of either sign; there are only longitudinal phonons in one dimension. Assuming a fixed sound speed $c_{s}$, the corresponding energy is $\varepsilon(p)=c_{s}|p|$. We calculate the maximum entropy by the approach of Sec.2.4. Eq.(11) gives the thermal entropy $s(p)$ of a mode at temperature $T$; this corresponds to maximum entropy for given mean energy. Integrating $s(p)$ with measure $L d p / 2 \pi \hbar$ from $p=-\infty$ to $p=+\infty$, integrating by parts, and rescaling we get

$$
H_{\max }=\frac{2 L k T}{\pi \hbar c_{s}} \int_{0}^{X} \frac{x d x}{e^{x}-1},
$$

where $X \equiv p_{\max } / k T$. There is a peak momentum because the continuum description of the medium through which the phonons propagate breaks down at the "lattice constant" $2 \varsigma$. Hence $X \approx \frac{\hbar c_{s}}{2 \varsigma k T}$. A similar calculation gives

$$
E=\frac{L(k T)^{2}}{\pi \hbar c_{s}} \int_{0}^{X} \frac{x d x}{e^{x}-1}
$$

for the thermal energy

In order for the continuum approximation to apply at all, we need $k T \gg \hbar c_{s} / L$ so that at least the lowest lying phonon levels be highly populated. If in addition $k T \ll \hbar c_{s} / \varsigma$ (both conditions can hold provided $N \gg 1$ ), we have $X \gg 1$ so that the upper limit in the integral can be extended to infinity; the integral then equals $\pi^{2} / 6$ (see Appendix B). Eliminating $k T$ between (165) and (166) leads to

$$
H_{\max }=\left(2 \pi E L / 3 \hbar c_{s}\right)^{1 / 2} ; \quad \hbar c_{s} / L \ll E \ll \hbar c_{s} L / \varsigma^{2} .
$$

This is reminiscent of Pendry's formula (16); however, the dependence on the signaling speed does not disappear here. In the limit of large temperature $X \ll 1$ so that we can replace the integral by $X$. We then get

$$
H_{\max } \approx L / \pi \varsigma ; \quad E \gg \hbar c_{s} L / \varsigma^{2},
$$

so that the information approches asymptotically a number a little smaller than the number of molecules in the chain. Evidently the thermal entropy never quite competes wuith the ground state entropy. Thus our earlier argument indicates that the information bound is always obeyed.

It will be noticed that inclusion of the rest energy of the substrate structure in the energy entering into the bound is crucial to the latter's correctness. The bound does not necessarily work if $E$ is taken as the excitation energy alone. It is thus interesting to study a case where there is no mass in the substrate. An example is information coded in the states of a scalar field confined to a one dimensional cavity of length $L$. A calculation analogous to the above gives

$$
H_{\max }=(2 \pi E L / 3 \hbar c)^{1 / 2} ; \quad E \gg \hbar c / L .
$$


This is the real information-storage analog of the Pendry formula (16). Does it obey the information bound? Yes. The constraint on $E$ guarantees that the argument of the square root is large. Thus $H_{\max }<2 \pi E L / 3 \hbar c$ so that bound (85) is obeyed. Of course, nothing has been proven about the range $E \lesssim \hbar c / L$. This case must be studied numerically by the microcanonical approach, as in Sec.5.3. The result is that for the full range of energies

$$
(H / E)_{\max }=0.216 L / \hbar c
$$

which is consistent with bound (85).

\section{The Spacetime View of Information}

Thus far, as customary in the field, we have treated information storage and communication as separate issues. But clearly they are not. A situation can be described purely in terms of information storage only in the rest frame of the storing device. In another Lorentz frame information flows with the motion of the device, and the communication facet surfaces. In fact, the Lorentz invariance of the laws of physics must mean that information storage and communication are inextricably linked, and proper understanding of one of them suffices for understanding of the other. Thus far no unified treatment of this sort exists. But there are some insights into how information is intertwined with the concept of spacetime. We describe these here.

\subsection{Influence of Uniform Motion on Communication}

One of our basic results in communication is that the information in a burst signal is bounded by Eq.(47). However, we never made it clear in what Lorentz frame one is to calculate $E$ and $\tau$. Normally transmitter and receiver are at rest in the same frame, and the question is not important. However, the transmitter can be in a spacecraft rapidly moving with respect to the earthbound receiver. Although the relative velocities in this example are nonrelativistic, it does illustrate that the question of Lorentz frame is not a trivial one.

However, it is easy to show that, under wide circumstances, Eq.(47) is a Lorentz invariant statement. For example, consider a "medium" such as a fluid or dielectric solid in which signals propagate with fixed speed $c_{s}$ and no dispersion. The carrier quanta could be phonons propagating in the fluid, or "dressed" photons propagating in a dielectric channel, etc. We assume there are no currents (flows) in the medium so that all of it is at rest in a given Lorentz frame A. Consider another Lorentz frame B moving to the right relative to A with speed $V$. Withouth loss of generality we may assume that their origins coincide at time $t_{A}=0$.

Let a right-moving signal's front pass the origin of A at that same time. We assume $V<c_{s}$; the opposite case can be studied with appropriate changes. At some time $t_{A}=t_{1}$ the signal's rear end will pass the origin of $A$, at which time the origin of $\mathrm{B}$ has reached position $x_{A}=V t_{1}$. At some later time $t_{A}=t_{2}$ the signal's rear has caught up with the origin of $\mathrm{B}$ which is then at $x_{A}=V t_{2}$. Calculating entirely in A we find $\left(c_{s}-V\right) t_{2}=c_{s} t_{1}$ so that

$$
t_{2} / t_{1}=\left(1-V / c_{s}\right)^{-1}
$$

Evidently, the duration of the signal in $\mathrm{A}$ is just $\tau_{A}=t_{1}$. Because of time dilation, the duration in $\mathrm{B}$ is just $\tau_{B}=t_{2} \gamma^{-1}$ where $\gamma \equiv\left(1-V^{2} / c^{2}\right)^{-1 / 2}$ is the Lorentz factor between the frames. Then by virtue of (142) we have

$$
\tau_{B}=\tau_{A}\left(1-V / c_{s}\right)^{-1} \gamma^{-1}
$$

Let us now look at the energy. If in A the energy and momentum of a quantum are $\epsilon$ and $p$, respectively, then by virtue of the constancy of the propagation velocity, $\epsilon=c_{s} p$. In the absence of interactions the total energy $E_{A}$ and momentum of the signal must stand in the same ratio Therefore, by the Lorentz transformation of energy and momentum, the signal energy in frame B is

$$
E_{B}=\gamma E_{A}\left(1-V / c_{s}\right)
$$

We now see from (172)-(173) that $E_{A} \tau_{A}=E_{B} \tau_{B}$ which shows that the quantity $\xi \equiv E \tau / \hbar$ is the same in the propagation medium's frame and in some other frame, eg that of the receiver in motion with respect to the medium. It is possible to demonstrate the invariance when B is the transmitter's frame by having frame B move to the left, and the signal to the right, with respect to A. Of course, the information $I$ is itself a Lorentz invariant. The end result is that the formula $I_{\max }=\Im(E \tau / \hbar)$ is Lorentz invariant. In particular, it has the same form in the frames of the medium (if other than vacuum), the transmitter, and the receiver.

When the signal moves precisely with the speed of light, e.g. photons in empty space, ..., the above argument may be rephrased by taking A as the transmitter's frame, while B is some other frame, like 
the receiver's. The calculations go through formally as before, and demonstrate the Lorentz invariance of $I_{\max }=\Im(E \tau / \hbar)$ in this case also.

\subsection{Influence of Gravitation on Communication}

Up to now we have implicitly assumed that the signal propagates in flat spacetime (no gravitational field). Consider now its propagation through an external stationary gravitational field, e.g. signaling from the surface of a planet to an orbiting spacecraft, or its propagation in the expanding universe (time dependent but spatially homogeneous gravitational field). In either case we assume the transmitter and receiver to be at rest (in the cosmological example this means at rest in the frame of the microwave background). Redshift effects will make the $E$ and $\tau$ at reception differ from those at transmission. However, $E \tau$ will be the same. To verify this focus on a single Fourier component of the wavepacket representing a particular signal state (in the cosmological case we refer to spatial Fourier component). Evidently, the variation of the phase from front to the rear of the packet must be conserved in transit. At a fixed point in the transmitter's frame A, the overall phase variation is just $\omega_{A} \tau_{A}$ where $\omega_{A}$ is the angular frequency or time derivative of the phase in frame A. Analogously at a fixed point in the receiver's frame B, the change of phase amounts to $\omega_{B} \tau_{B}$. Now for a single quantum $\epsilon=\hbar \omega$. Therefore, if field self-interaction may be neglected ( $E$ is the sum of $\epsilon$ 's), if the signal transit is adiabatic (no quantum transitions between various states), and if dispersion is absent (signal does not spread), then $E \tau$ will be conserved in transit. The same adiabaticity assumption guarantees that information is not lost. Thus formula (47) is equally valid as applied to transmitter or receiver (or in any motionless frame in between).

By combining this result with our previous one on Lorentz invariance interpreted locally, we conclude that $I_{\max }=\Im(E \tau / \hbar)$ must be valid in all Lorentz frames, and in the presence of external stationary or time dependent but spatially homogeneous gravitational fields.

The previous argument neglected the self-gravitation of the signal: the gravitational field was taken as external. Although in everyday signals self-gravitation is indeed negligible, the issue of self-gravitating signals is of great intrinsic interest. Once self-gravitation is present, Newton's constant $G$ enters into the discussion alongside $\hbar$ and $c$. Reviewing the argument developed in Sec.3.1., and excluding again lengths derived from Compton lengths or frequency cutoffs, we conclude that there are now two independent dimensionless combinations of $E, \tau$ and natural constants. These can be taken as $\xi=E \tau / \hbar$ and $\varpi \equiv G E c^{-5} \tau^{-1}$. Thus the CIF must be a function of $\xi$ and $\varpi: I_{\max } \equiv \Im(\xi, \varpi)$.

The parameter $\varpi$ is of the order of the ratio of the gravitational binding energy of the signal to the signal energy $E$ and, therefore, a good measure of self-gravitation. This assumes that the size of the signal is $c \tau$; if the propagation speed $c_{s}$ is smaller than $c, \varpi$ is a lower bound on the specific gravitational binding energy. Another interpretation: $\varpi$ is the ratio of the Schwarzschild radius of the signal $G E / c^{4}$ to its size $c \tau$ (if $c_{s}<c, \varpi$ is only a lower bound on the ratio). From these comments it is clear that $\varpi$ has a maximum value of order unity. When $\varpi \ll c_{s} / c$, self-gravitation is negligible, and the CIF reduces to a function of $\xi$ only, as in Sec.3.

When $\varpi$ is maximal, the signal collapses into a black hole (signal has shrunk down to its Schwarzschild radius), and it can longer convey any information. Thus $\Im \rightarrow 0$ in that limit. It thus seems likely that the main effect of nonnegligible $\varpi$ is to reduce the CIF below its value for $\varpi=0$. As of yet no calculation of the dependence on $\varpi$ has been made.

\subsection{Acceleration as a Communication Jammer}

In Sec.6.1. we saw that communication between transmitter and receiver in motion with respect to each other can be described with the same CIF as for transmitter and receiver at rest. What if the motion is accelerated? We might be tempted to argue that momentarily the transmitter and receiver are related by a particular Lorentz transformation, so that the description via the Lorentz invariant CIF can be employed. However, this line of reasoning leaves out a crucial point of principle. As discovered by Unruh, ${ }^{64}$ a receiver moving with uniform acceleration $a$ (this is a statement independent of Lorentz frame) is subject to quantum noise having all the properties of thermal radiation with temperature $T_{U}=\hbar a / 2 \pi c k$. Any communication with that receiver is thus affected by thermal noise intrinsically connected with its motion.

Let us, for simplicity, consider communication in the limit of very long duration signals. The relevant formalism is Lebedev and Levitin's for a broadband noisy channel (see Sec.2.5). We recall that the argument is concerned mainly with the receiver. The channel capacity Eq.(25) is governed by one parameter, the 
power $P$ received. Now power is a Lorentz invariant ${ }^{65}$. Therefore, for a steady state transmitter, the $P$ received is also constant although the receiver is constantly changing its speed. Let us make the substitution $k T_{1} \rightarrow \hbar a / 2 \pi c$ in Eq.(25). We get

$$
\dot{I}_{\max }=\frac{a}{12 c}\left\{\left[1+\frac{48 \pi c^{2} P}{\hbar a^{2}}\right]^{1 / 2}-1\right\} \log _{2} e \text { bits s}^{-1} .
$$

For large $P(174)$ goes over to the Pendry formula (16). For low $P$ we have

$$
\dot{I}_{\max } \approx(2 \pi c P / \hbar a) \log _{2} e \text { bits s}^{-1} \text {. }
$$

The transition occurs at a characteristic power $P_{c}=10^{-2} \hbar a^{2} c^{-2}$. Although for everyday accelerations this is a tiny power, for the acceleration typical of electrons in atoms $\left(10^{25} \mathrm{~cm} \mathrm{~s}^{-2}\right), P_{c} \sim 10^{12} \mathrm{ev} \mathrm{s}^{-1}$ which is quite large. It thus maybe that the transfer of information to and from elementary particles involved in natural processes is governed primarily by the limiting form (175).

\section{Acknowledgments}

JDB thanks John A. Wheeler for suggestions. MS thanks Jamil Daboul for comments, and the Physics Department, Ben-Gurion University for hospitality. Partial support by a grant from the Wolf Foundation for the Advancement of Science and Art administered by the Israel National Academy of Sciences is acknowledged.

\section{References}

1. H. Nyquist, Bell Syst. Tech. J., April (1924) 324; H. Nyquist, Trans. A.I.E.E 47 (1928) 617; R. V. L. Hartley, Bell Syst. Tech. J., July (1928) 535.

2. C. Shannon and W. Weaver, The Mathematical Theory of Communication, (Univ. of Illinois Press, 1949).

3. T. E.Stern, IEEE Trans. Inf. Theory IT-6 (1960) 435.

4. J. P.Gordon, Proc.IRE 50 (1962) 1898.

5. J. P. Gordon, in Advances in Quantum Electronics, ed. J. R. Singer, (Columbia University Press, 1961).

6. H. Marko, Kybernetik 2 (1965) 274.

7. D. S. Lebedev and L. B. Levitin, Dokl. Akad. Nauk SSSR 149 (1963) 1299 [Sov. Phys. Dokl. 8 (1963) 377].

8. J. B. Pendry, J. Phys. A16 (1983) 2161.

9. H. J. Bremermann, in Self-Organizing Systems, eds. M. C. Yovitz, T. C. Jacobi and G. D. Goldstein, (Spartan Books, 1962).

10. H. J. Bremermann, in Proc. Fifth Berkeley Symp. on Mathematical Statistics and Probability, eds. L. M. LeCam and J. Neyman, (Univ. of California Press, 1967).

11. H. J. Bremermann, Int. J. Theor. Phys. 21 (1982) 203.

12. J. D. Bekenstein, Phys. Rev. Letters 46 (1981) 623.

13. J. D. Bekenstein, Phys. Rev. D 30 (1984) 1669.

14. R. Landauer, Int. J. Theor. Phys. 21 (1982) 283.

15. J. D. Bekenstein, Phys. Rev. A 37 (1988) 3437.

16. J. D. Bekenstein, Phys. Rev. D 7 (1973) 2333.

17. J. D. Bekenstein, Phys. Rev. D 9 (1974) 3292.

18. S. W. Hawking, Commun. Math. Phys. 43 (1975) 199.

19. J. D. Bekenstein, Phys. Rev. D 23 (1981) 287.

20. A. Qadir, Phys. Lett. 95A (1983) 285.

21. I. Kahn and A. Qadir, Lett. Nuov. Cim. 41 (1984) 493.

22. J. D. Bekenstein and E. I. Guendelman, Phys. Rev. D 35 (1987) 716.

23. M. Schiffer and J.D. Bekenstein, Phys. Rev. D 39 (1989) 1109.

24. Y. Yamamoto and H. A. Haus, Revs. Mod. Phys. 58 (1986).

25. H. Takahashi, in Advances in Communication Systems, ed. A. V. Balakrishnan, (Academic Press, 1965).

26. B. Saleh and M. Teich, Phys. Rev. Letters 58 (1987) 2656.

27. R. Landauer, Phys. Scripta 35 (1987) 88; R. Landauer, Ann. N. Y. Acad. Sci. 426 (1984) 161; R. Landauer, Found. Phys. 16 (1986) 551.

28. L. Szilard, Z.Phys. 53 (1929) 840.

29. L. Brillouin, Science and Information Theory, (Academic Press, 1965).

30. E. Jaynes, Phys. Rev.106 (1957) 620; E. Jaynes, Phys. Rev.108 (1957) 171.

31. A. Katz, Principles of Statistical Mechanics: The Information Theory Approach, (Freeman, 1967).

32. G. Chaitin, Algorithmic Information Theory, (Cambridge University Press, 1987).

33. L. D. Landau and E. M. Lifshitz, Statistical Physics, Part II, (Pergamon, 1980), p.326. 
34. L. D. Landau and E. M. Lifshitz, Statistical Physics, Part I, 3rd ed., (Pergamon, 1980).

35. D. Gabor, Phil. Mag. 41 (1950) 7, 1161.

36. E. Merzbacher, Quantum Mechanics, (Wiley, 1970).

37. M. Schiffer, "Shannon's Information is not Entropy", submitted to Phys. Lett.A, 1990.

38. L. D. Landau and E. M. Lifshitz, Quantum Mechanics, 3rd. ed., (Pergamon, 1977).

39. E. P. Wigner, in Aspects of Quantum Theory, eds. A. Salam and E. P. Wigner, (Cambridge University Press, 1972).

40. M. Schiffer, "The Quantum Limit for Information Transmission", submitted to Phys. Lett.A, 1990.

41. R. Landauer and J. W. F. Woo, in Synergetics, ed. H. Haken, (Teubner, 1973).

42. G. Gibbons (1980), unpublished.

43. L. B. Levitin, Int. J. Theor. Phys. 21 (1982) 299.

44. J. D. Bekenstein, Phys. Rev. D 27 (1983) 2262.

45. W. Lukosz, Z. Phys. 262 (1973) 327.

46. S.D. Unwin, Phys. Rev. D 26 (191982) 944.

47. J. Ambjorn and S. Wolfram, Ann. Phys. 147 (1983) 1.

48. D. Page, Phys. Rev. D 26 (1982) 947.

49. D. Deutsch, Phys. Rev. Letters 48 (1982) 286.

50. J. W. Dettman, Mathematical Methods in Physics and Engineering, (McGraw-Hill, 1962).

51. M. Abramowitz and I. Stegun, Handook of Mathematical Functions, (Dover, 1965).

52. M. Schiffer and J.D. Bekenstein, Phys. Rev. D 42 (1990), in press.

53. W. G. Unruh, Phys. Rev. D 42 (1990), in press.

54. W. G. Unruh and R. M. Wald, Phys. Rev. D 25 (1982) 942.

55. K. H. Mariwalla (1981), unpublished.

56. J. D. Bekenstein, General Relativity and Gravitation 14 (1982) 355.

57. R. Hagedorn, Nuov. Cim. 56A (1968) 1027; R. Hagedorn, Astron. Astrophys. 5 (1970) 184.

58. K. Huang and S. Weinberg, Phys. Rev. Letters 25 (1970) 895.

59. R. Rajaraman, Physics Reports 21 (1975) 239.

60. R. F. Dashen, B.Hasslacher and A. Neveau, Phys. Rev. D 10 (1974) 4130.

61. M. Schiffer, Dissertation, Ben Gurion University (1988).

62. R. Sorkin, R. M. Wald and Z. Z. Jiu, General Relativity and Gravitation 13 (1981) 1127.

63. W. H. Zurek and D. N. Page, Phys. Rev. D 29 (1984) 628.

64. W. G. Unruh, Phys. Rev. D 14 (1976) 870.

65. J. D. Jackson, Classical Electrodynamics, (Wiley, 1962).

66. I. S. Gradshteyn and I. M. Ryzhik, Table of Integrals, Series and Products, (Academic Press, 1980).

\section{Appendix A}

Here we prove Theorem 1. First we write the composition law

$$
\left(1-e^{-\beta}\right) e^{-\beta m}=\left(1-e^{-\alpha}\right) \sum_{n=0}^{m} e^{-\alpha(m-n)} Q(n) .
$$

In (A.1) we now shift $m \rightarrow m+1$, multiply the equation by $e^{\beta}$ and substract from the original equation. Separating out the term of index $m+1$, and replacing the remaining sum over $n$ by means of Eq.(A.1), we are able to solve for

$$
Q(m+1)=\frac{1-e^{-\beta}}{1-e^{-\alpha}}\left(1-e^{\beta-\alpha}\right) e^{-\beta(m+1)} .
$$

This agrees with Eq.(46) for $m \neq 0$. To finish the proof, we consider the $m=0$ case of Eq.(A.1). By virtue of $n$ being fixed as 0 , we immediately get the $m=0$ case of Eq.(46) $\square$.

\section{Appendix B}

Here we prove Eqs. (64)-(65). The Euler Maclaurin summation formula with the residue term left out is

$$
\sum_{n=1}^{N-1} f(n)=\int_{1}^{N} f(x) d x+\frac{f(1)+f(N)}{2}+\left.\frac{1}{2 !} B_{2} f^{(1)}(x)\right|_{1} ^{N}+\left.\frac{1}{4 !} B_{4} f^{(3)}(x)\right|_{1} ^{N}+\ldots
$$

where $B_{n}$ are the Bernoulli numbers: $B_{0}=1, B_{1}=-\frac{1}{2}, B_{2}=\frac{1}{6}$ and $B_{p}=0$ for $p=3,5,7, \ldots \quad$ Suppose $f(x)$ is such that it and all its derivatives vanish for large arguments. Let us take $N \rightarrow \infty$. In such a situation Eq.(B.1) may be cast in the form

$$
\sum_{1}^{\infty} f(n)=\int_{0}^{\infty} f(x) d x-\int_{0}^{1} f(x) d x-\sum_{p=1}^{\infty} \frac{1}{p !} B_{p} f^{(p-1)}(1)
$$


We shall apply this summation formula to the function $f(x)=x\left(e^{\beta x}-1\right)^{-1}$ which satisfies the mentioned conditions. We first perform the integral

$$
\int_{0}^{\infty} f(x) d x=\beta^{-2} \int_{0}^{\infty} \frac{x d x}{e^{x}-1}=\frac{\pi^{2}}{6 \beta^{2}}
$$

Keeping in mind that $f(x)$ is the generating function of the Bernoulli numbers, namely,

$$
f(x)=\frac{x}{\left(e^{\beta x}-1\right)}=\sum_{k=0}^{\infty} \frac{1}{k !} B_{k} x^{k} \beta^{k-1},
$$

we obtain for the second integral

$$
\int_{0}^{1} f(x) d x=\int_{0}^{1} \frac{x d x}{\left(e^{\beta x}-1\right)}=\beta^{-1} \sum_{k=0}^{\infty} \frac{1}{k+1 !} B_{k} \beta^{k} .
$$

By virtue of (B.4), we may express

$$
\sum_{p=1}^{\infty} \frac{1}{p !} B_{p} f^{(p-1)}(1)=\beta^{-1} \sum_{p=1}^{\infty} \sum_{k=p-1}^{\infty} \frac{1}{k+1 !} B_{k} \beta^{k}\left(\begin{array}{c}
k+1 \\
p
\end{array}\right) B_{p}
$$

Next with due care of the limits, we interchange the order of summation:

$$
\begin{gathered}
\sum_{p=1}^{\infty} \frac{1}{p !} B_{p} f^{(p-1)}(1)=\beta^{-1} \sum_{k=0}^{\infty} \sum_{p=1}^{k+1} \frac{1}{(k+1) !} B_{k} \beta^{k}\left(\begin{array}{c}
k+1 \\
p
\end{array}\right) B_{p} \\
=\beta^{-1}\left\{B_{0} B_{1}+\sum_{k=1}^{\infty} \frac{1}{(k+1) !} B_{k} \beta^{k} \sum_{p=1}^{k+1}\left(\begin{array}{c}
k+1 \\
p
\end{array}\right) B_{p}\right\} \\
=\beta^{-1}\left\{B_{0} B_{1}+\sum_{k=1}^{\infty} \frac{1}{(k+1) !} B_{k} \beta^{k}\left[\sum_{p=0}^{k+1}\left(\begin{array}{c}
k+1 \\
p
\end{array}\right) B_{p}-B_{0}\right]\right\} .
\end{gathered}
$$

Recalling that $B_{0}=1$ and $B_{1}=-\frac{1}{2}$ and using the identity ${ }^{51}$ between Bernoulli's numbers

$$
\sum_{p=0}^{k+1}\left(\begin{array}{c}
k+1 \\
p
\end{array}\right) B_{p}=B_{k+1}
$$

we write

$$
\sum_{p=1}^{\infty} \frac{1}{p !} B_{p} f^{(p-1)}(1)=\beta^{-1}\left\{-\frac{1}{2}+\sum_{k=1}^{\infty} \frac{1}{(k+1) !} B_{k} \beta^{k}\left(B_{k+1}-1\right)\right\} .
$$

Since $B_{k}=0$ for $k=3,5, \ldots, B_{k} B_{k+1}=0$ for $k \geq 2$. Inserting (B.3), (B.5) and (B.9) into (B.2) we obtain finally

$$
\sum_{j=1}^{\infty} \frac{j}{e^{\beta j}-1} \approx \frac{\pi^{2}}{6 \beta^{2}}-\frac{1}{2 \beta}+\frac{1}{24} .
$$

Since the derivative with respect to $\beta$ of $\ln Z$ in Eq.(62) is the negative of the sum in (B.10), $\ln Z$ may be obtained by integration of the former expression with respect to $\beta$. The integration constant was obtained by performing the sum (62) numerically for, say, $\beta=1$. We find

$$
\ln Z \approx \frac{\pi^{2}}{6 \beta}+\frac{1}{2} \ln \beta-\frac{\beta}{24}-0.91894 .
$$

\section{Appendix C}

Here we establish the integral equation (106). First, since $\frac{d n}{d E}$ is a sum of delta functions [see Eq.(100)], we have for $m \geq 1$ the identity

$$
N_{m}(E)=\int_{0}^{E} d E_{1} \int_{0}^{E} d E_{2} \ldots \int_{0}^{E} d E_{m-1}
$$




$$
\left(\frac{d n}{d E_{2}}\right) \ldots\left(\frac{d n}{d E_{m-1}}\right) n\left(E-\sum_{k=1}^{m-1} E_{k}\right) .
$$

This equation may be put in an equivalent form by recalling that the function $n\left(E-\sum_{k=1}^{m-1} E_{k}\right)$ vanishes for a negative argument, i.e., the inequality $E_{p} \leq E-\sum_{k=1}^{p-1} E_{k}$ is always satisfied. Thus

$$
\begin{aligned}
N_{m}(E)= & \int_{0}^{E} d E_{1} \int_{0}^{E-E_{1}} d E_{2} \ldots \int_{0}^{E-E_{1}-E_{2} \ldots-E_{m-2}} d E_{m-1} \\
& \left(\frac{d n}{d E_{1}}\right)\left(\frac{d n}{d E_{2}}\right) \ldots\left(\frac{d n}{d E_{m-1}}\right) n\left(E-\sum_{k=1}^{m-1} E_{k}\right)
\end{aligned}
$$

for $m \geq 1$ together with Eq.(103). Putting these pieces together, $N(E)$ is expressable as

$$
\begin{gathered}
N(E)=\Theta(E)+\sum_{m=1}^{\infty} \int_{0}^{E} d E_{1} \int_{0}^{E-E_{1}} d E_{2} \ldots \int_{0}^{E-E_{1}-E_{2} \ldots-E_{m-2}} d E_{m-1} \\
\left(\frac{d n}{d E_{1}}\right)\left(\frac{d n}{d E_{2}}\right) \ldots\left(\frac{d n}{d E_{m-1}}\right) n\left(E-\sum_{k=1}^{m-1} E_{k}\right) .
\end{gathered}
$$

This messy expression is nothing but the iteration of the integral equation

$$
N(E)=\Theta(E)+\int_{0}^{E} N\left(E-E^{\prime}\right)\left(\frac{d n}{d E^{\prime}}\right) d E^{\prime}
$$

starting from $N(E)=n(E)$. Eq.(C.4) is identical to Eq.(106).

\section{Appendix D}

Here we use the method of Ref.40 to evaluate the integral

$$
N(E)=\frac{1}{2 \pi i} \int_{\gamma-i \infty}^{\gamma+i \infty} \frac{e^{\epsilon S}-1}{s\left(e^{\epsilon S}-2\right)} e^{s E} d s .
$$

Define the variables $\sigma \equiv \epsilon s-\ln 2$ and $a \equiv E / \epsilon$. The above expression then reads

$$
N(E)=2^{a} I(a),
$$

where

$$
I(a) \equiv \frac{1}{2 \pi i} \int_{\gamma \epsilon-i \infty}^{\gamma \epsilon+i \infty} \frac{\left(2 e^{\sigma}-1\right) e^{a \sigma}}{2(\sigma+\ln 2)\left(e^{\sigma}-1\right)} d \sigma .
$$


Now push the contour leftwards to minus infinity while indenting it so as not to overrun any of the infinity of poles $\sigma=i 2 \pi k$ with $k$ integral, as shown in Fig.5. (note that there is no pole at $\sigma=-\ln 2$ ). By Cauchy's theorem the integral is

$$
I(a)=\sum_{k=-\infty}^{\infty} \frac{e^{i 2 \pi a k}}{2(i 2 \pi k+\ln 2)} .
$$

At this point we expand the exponential in sines and cosines and rationalize the complex denominator. Four series result of which two vanish by symmetry. We are left with

$$
I(a)=\frac{1}{2 \ln 2}+\frac{\eta^{2}}{\ln 2} \sum_{k=1}^{\infty} \frac{\cos (2 \pi a k)}{k^{2}+\eta^{2}}+\frac{1}{2 \pi} \sum_{k=1}^{\infty} \frac{k \sin (2 \pi a k)}{k^{2}+\eta^{2}}
$$

where $\eta \equiv \ln 2 / 2 \pi$. Notice that $I$ depends only on the fractional part of its argument because addition of any integer to $a$ leaves the above series unchanged.

Now recall the identities ${ }^{66}$

$$
\sum_{k=1}^{\infty} \frac{\cos (k x)}{k^{2}+\eta^{2}}=\frac{\pi}{2 \eta} \frac{\cosh \eta(\pi-x)}{\sinh \eta \pi}-\frac{1}{2 \eta^{2}} ; \quad 0 \leq x \leq 2 \pi .
$$

and

$$
\sum_{k=1}^{\infty} \frac{k \sin (k x)}{k^{2}+\eta^{2}}=\frac{\pi}{2} \frac{\sinh \eta(\pi-x)}{\sinh \eta \pi} ; \quad 0 \leq x \leq 2 \pi .
$$

Expanding the hyperbolic functions, using the explicit value of $\eta$, and setting $x=2 \pi a$, we can use these to reduce (D.5) to the form

$$
I(a)=2^{-[a]},
$$

where $[a]$ stands for the fractional part of $a$. The whole part of $a$ drops out for the reason mentioned above. Going back to (D.2) we see that $N(E)$ is 2 to the whole part of $a$. 
This figure "Fig1.jpg" is available in "jpg" format from: http://arxiv.org/ps/quant-ph/0311050v1 
This figure "Fig2.jpg" is available in "jpg" format from: http://arxiv.org/ps/quant-ph/0311050v1 
This figure "Fig3.jpg" is available in "jpg" format from: http://arxiv.org/ps/quant-ph/0311050v1 
This figure "Fig4.jpg" is available in "jpg" format from: http://arxiv.org/ps/quant-ph/0311050v1 
This figure "Fig5.jpg" is available in "jpg" format from: http://arxiv.org/ps/quant-ph/0311050v1 